\title{
Generation of 100-year geomagnetically induced current scenarios
}

\author{
A. Pulkkinen, ${ }^{1,2}$ E. Bernabeu, ${ }^{3}$ J. Eichner, ${ }^{4}$ C. Beggan, ${ }^{5}$ and A. W. P. Thomson ${ }^{5}$
}

Received 15 November 2011; revised 21 February 2012; accepted 22 February 2012; published 24 April 2012.

[1] A series of 100-year extreme geoelectric field and geomagnetically induced current (GIC) scenarios are explored by taking into account the key geophysical factors associated with the geomagnetic induction process. More specifically, we derive explicit geoelectric field temporal profiles as a function of ground conductivity structures and geomagnetic latitudes. We also demonstrate how the extreme geoelectric field scenarios can be mapped into GIC. Generated statistics indicate $20 \mathrm{~V} / \mathrm{km}$ and $5 \mathrm{~V} / \mathrm{km}$ 100-year maximum 10-s geoelectric field amplitudes at high-latitude locations with poorly conducting and well-conducting ground structures, respectively. We show that there is an indication that geoelectric field magnitudes may experience a dramatic drop across a boundary at about $40^{\circ}-60^{\circ}$ of geomagnetic latitude. We identify this as a threshold at about $50^{\circ}$ of geomagnetic latitude.

The sub-threshold geoelectric field magnitudes are about an order of magnitude smaller than those at super-threshold geomagnetic latitudes. Further analyses are required to confirm the existence and location of the possible latitude threshold. The computed extreme GIC scenarios can be used in further engineering analyses that are needed to quantify the geomagnetic storm impact on conductor systems such as high-voltage power transmission systems. To facilitate further work on the topic, the digital data for generated geoelectric field scenarios are made publicly available.

Citation: Pulkkinen, A., E. Bernabeu, J. Eichner, C. Beggan and A. W. P. Thomson (2012), Generation of 100-year geomagnetically induced current scenarios, Space Weather, 10, S04003, doi:10.1029/2011SW000750.

\section{Introduction}

[2] The potential for severe societal consequences has been driving recent interest in extreme geomagnetic storm impacts particularly on high-voltage power transmissions systems [National Research Council, 2008; North American Electric Reliability Corporation and the Department of Energy, 2010]. Although GPS-based timing of the transmission system operations can be impacted by space weather storms, it is generally understood that geomagnetically induced currents (GIC), causing half-cycle saturation of high-voltage power transformer are the leading mode for the most severe problems, such as electric blackouts and equipment damage [Kappenman, 1996; Molinski, 2002]. Consequently, characterization of extreme GIC events is central for quantifying the technological impacts and societal consequences of extreme space weather.

[3] In this paper we investigate the general characteristics of extreme geoelectric field and GIC events. The

\footnotetext{
${ }^{1}$ The Catholic University of America, Washington, D.C., USA.

${ }^{2}$ NASA Goddard Space Flight Center, Greenbelt, Maryland, USA.

${ }^{3}$ Dominion Virginia Power, Richmond, Virginia, USA.

${ }^{4}$ Munich Re, Munich, Germany.

${ }^{5}$ Geomagnetism Team, British Geological Survey, Edinburgh, UK.
}

geoelectric field induced in the ground by spatiotemporally varying magnetospheric and ionospheric electric current systems is the primary physical quantity driving GIC and often a simple linear relationship is sufficient for mapping geoelectric field into GIC [Viljanen et al., 2006a; Pulkkinen et al., 2006; Ngwira et al., 2008; Pulkkinen et al., 2010]. Thus, the key challenge is to characterize extreme geoelectric field events, which is the primary goal of the paper.

[4] Complete analysis of the risk from extreme space weather impacts on, for example, high-voltage power transmissions systems also requires engineering analyses, specifying how given extreme GIC impact the performance of the transformers and the system as a whole. Such a holistic definition of risk goes beyond the pure probability of occurrence of strong GIC events of certain magnitudes. It also comprises aspects of the vulnerability, i.e., how susceptible or robust today's power transmission systems are to the physical GIC hazard. In addition, depending on parameters such as the geographical location or the time of day or the season, the impact of the same physically extreme (and hence rare) event might produce a very different outcome. Such improved concepts are widely used in the natural catastrophe risk modeling community [Grossi and Kunreuther, 2005], e.g. in the insurance sector. However, today's natural catastrophe 
risk models have their main focus on more prevalent hazards, such as meteorological, hydrological events and geophysical events (e.g. hurricanes and earthquakes) [Muir-Wood and Grossi, 2008]. Although more detailed engineering analyses are out of scope of the work at hand, our purpose is to facilitate further engineering and hazard analyses quantifying the risk extreme geomagnetic storms pose on high-voltage power transmission.

[5] The geomagnetic induction process that generates the ground geoelectric field is dependent both on the characteristics of geospace electric currents and on the local geological conditions which dictates the electromagnetic response of the medium to geospace driving [Wait, 1970; Berdichevsky and Zhdanov, 1984; Weaver, 1994, for classic treatments]. Consequently, the geoelectric field is a complex function of a number of geophysical factors that all need to be accounted for in the extreme event analysis. The key factors to consider are: the effect of the ground conductivity structure on the extreme geoelectric field amplitudes; the effect of the geomagnetic latitude on the extreme geoelectric field amplitudes; temporal scales of the extreme geoelectric fields; and spatial scales of the extreme geoelectric fields.

[6] Due to the lack of observational information about extreme events and due to the great variety of, for example, local geological conditions, accounting for all four factors above is a substantial challenge. Clearly, approximations and extrapolations are required in the analysis and the question thus becomes "what is the most feasible practical approach that also provides information directly usable in further engineering analyses?" These considerations lead us to use an extreme event scenario approach. In this approach our goal is to generate several scenarios that represent the variability of the extreme events as a function of the four factors above. Further, the scenario approach will provide representative GIC time series that can be used directly in further engineering analyses.

[7] Another question that must be addressed prior to our analyses is "what constitutes an extreme event?" Extreme events can be defined in a number of different ways. For example, there has been discussion in the GIC community that one should look, for example, at "10 times March 13, 1989 event." In this case the date 13 March 1989 refers to the space weather event that led to the collapse of the Hydro Quebec high-voltage power transmissions system in Canada [Bolduc, 2002]. However, "10 times the March 13, 1989 event" is not a rigorous definition for an extreme event. One first needs to define what physical parameters are used to amplify the 13 March 1989 event tenfold. If one of the parameters is, for example, the Dst index (classic parameter used in measuring the geomagnetic storm magnitude), one is immediately faced with another problem. Namely, the minimum Dst index of the 13 March 1989 was $-589 \mathrm{nT}$ while the minimum Dst of the Carrington storm event of 1-2 September 1859 has been estimated to be approximately $-850 \mathrm{nT}$ [Siscoe et al., 2006]. The Dst estimate by Siscoe et al. [2006] is based on hourly averages mimicking the generation of the standard Dst index. The maximum low-latitude excursion during the Carrington event was approximately $1600 \mathrm{nT}$ [Tsurutani et al., 2003]. The Carrington geomagnetic storm event is the largest in the recorded history and the minimum Dst of the event is only about $45 \%$ larger in absolute magnitude than Dst of the 13 March 1989 event. Consequently, amplifying the March 1989 event tenfold in terms of Dst index magnitude would quickly lead to unrealistic extreme storm scenarios. We note that a similar argumentation applies also for the time derivative of the magnetic field often used as an indicator for GIC activity [Viljanen et al., 2001]. First, there is no rigorous justification for arbitrarily amplifying the largest magnetic field fluctuations of the March 1989 event tenfold. Second, despite the often good statistical association, the time derivative of the magnetic field is not the primary physical quantity driving GIC and consequently there is no direct one-toone relation between the two parameters.

[8] We will instead use a rigorous statistical definition for an extreme event and select a physical parameter that is directly related to GIC. More specifically, we define an extreme event as the maximum 100-year amplitude of the 10-s resolution horizontal geoelectric field. The details of the definition will be discussed more in detail below but the basic philosophy for selecting a 100-year event is quite simple: we are looking for extreme events that occur significantly less frequently than once per solar cycle (i.e., 11 years), while at the same time being careful not to extrapolate the available observational information and statistics too far. As will be shown below, extrapolating the statistics to a 100-year event is still reasonable, while extracting information about significantly rarer events may not be feasible.

[9] Section 2 of the paper describes the process used for generating the 100-year geoelectric field scenarios. We first discuss the general philosophy of the extreme scenario approach in detail and then, in section 2.1, describe the generation of the baseline statistics. Subsequent sections describe the analyses associated with the four different factors: section 2.2 addresses the effect of the ground conductivity structure on the extreme amplitudes; section 2.3 discusses the effect of the geomagnetic latitude on the extreme amplitudes; section 2.4 describes the temporal scales of the extreme events; and section 2.5 discusses the spatial scales of the extreme events. Section 3 summarizes the generated extreme geoelectric field scenarios. In section 4 we describe how the geoelectric field scenarios can be mapped into GIC, and section 5 provides further discussion and outlines some of the work that will be required to refine and improve the scenarios generated in this paper.

\section{Generation of Extreme Geoelectric Field Scenarios}

[10] As explained above, we selected the extreme event scenario approach to account for the variability of geospace and geological conditions associated with extreme 
GIC events. More specifically, scenarios will be derived as a function of different representative ground conductivity structures and geomagnetic latitudes. Typical temporal scales of storm events will be captured by using temporal profiles from a representative storm event. One hundredyear scenarios are then achieved by scaling the representative storm event by the maximum amplitudes obtained via extrapolation of the geoelectric field amplitude statistics. In other words, we generate an artificial storm event that will produce the 100-year peak amplitudes.

[11] We chose to scale an actual observed storm instead of using synthetic temporal profiles because of two major reasons. First, as will be discussed more in detail below, there are many types of dynamical processes in the solar wind-magnetosphere-ionosphere system that are capable of generating large GIC. All of these processes have their distinct spectral characteristics and consequently no single simple synthetic temporal profile is capable of capturing the full variability observed during extreme storms. Second, the selected representative storm profiles instead include actual spectral signatures of many relevant geospace drivers of large GIC. Using actual observed storm events also captures the length of the extreme storms, which may be important from the engineering analysis viewpoint.

[12] It is important to note that the linear scaling of representative storm event carried out in this work is supported by the statistical results of Weigel and Baker [2003] and Pulkkinen et al. [2008]. Weigel and Baker [2003] found that the shape of the probability distribution of high-latitude ground magnetic field fluctuations is nearly independent of solar wind driving conditions. Because the average solar wind state primarily enters through the standard deviation of the distributions derived by Weigel and Baker [2003], the solar wind input can be viewed as a linear amplifier of the high-latitude ground magnetic field fluctuations. In other words, one can select an arbitrary geomagnetic storm event and scale it to represent different solar wind driving conditions. Plane wave-based mapping of the horizontal ground magnetic field components into the geoelectric field is a linear operation (see Appendix A), which has been shown to produce accurate modeled GIC. It follows that the results of Weigel and Baker [2003] also hold for the (modeled) geoelectric field.

[13] On the other hand, Pulkkinen et al. [2008] studied the statistics of modeled geoelectric field amplitudes at highlatitude locations. They found that the probability distribution of the geoelectric field amplitudes is approximately lognormal and that the shape of the distribution is nearly independent of both the solar wind convective electric field amplitude and the magnetospheric state measured in terms of the Dst index. Further, the distribution was shifted monotonically as the solar wind or magnetospheric conditions became more disturbed. This feature can be understood by considering a lognormal probability density function of a random variable $X$

$$
p_{X}(x)=\frac{1}{x \sigma \sqrt{2 \pi}} e^{-\frac{(\ln x-\mu)^{2}}{2 \sigma^{2}}},
$$

where $\mu$ and $\sigma$ are the location and scale of the function, respectively. Linear amplification of the variable $X$ by a factor $\alpha$, equals to change of variable $Y=g(X)=\alpha X$. The new density function under change of variable can be calculated from

$$
p_{Y}(y)=\frac{1}{\left|g^{\prime}\left(g^{-1}(y)\right)\right|} p_{X}\left(g^{-1}(y)\right),
$$

where $g^{\prime}$ indicates derivative of the function $g$. Equations (1) and (2) then give under linear transformation $Y=\alpha X$

$$
p_{Y}(y)=\frac{1}{|\alpha|} p_{X}\left(\frac{y}{\alpha}\right)=\frac{1}{y \sigma \sqrt{2 \pi}} e^{-\frac{(\ln y-\mu-\ln \alpha)^{2}}{2 \sigma^{2}}}
$$

In other words, the location of the lognormal distribution gets shifted $\mu \rightarrow \mu+\ln \alpha$. It follows that in terms of the shift of the lognormal distribution, the solar wind convective electric field and the Dst index can be viewed as a linear amplifier of the modeled high-latitude geoelectric field fluctuations. This finding is in a good agreement with the results by Weigel and Baker [2003] discussed above. Consequently, there is a good statistical justification for linear scaling of the geoelectric field to represent the most extreme solar wind conditions responsible for the most extreme GIC.

\subsection{Generation of the Statistics}

[14] The basis of the statistical analysis is identical to that of Pulkkinen et al. [2008]. We also note that Campbell [1980], Langlois et al. [1996], and Boteler [2001], among others, have studied the statistical aspects of extreme GIC events, but their studies used much more limited data sets or relied on empirical relations with geomagnetic indices. In this work the statistics are generated by using 10-s geomagnetic field recordings from 23 high-latitude IMAGE magnetometer chain sites for the period of January 1993 to December 2006. High quality final IMAGE data (for a description of quality control procedures, see http://space.fmi.fi/image/ accuracy.html) used in the study is also highly continuous and short data gaps no longer than few minutes were patched using linear interpolation. It is noted that the data covers one solar cycle period, which should make the extrapolation of the statistics to 100-year amplitudes more representative. The IMAGE stations are located in Northern Europe and cover about $55^{\circ}-75^{\circ}$ of geomagnetic latitude (corrected geomagnetic coordinates). Geomagnetic data from each IMAGE station are used to compute the local geoelectric field magnitudes $E=|E|$, where $E$ is the horizontal geoelectric field. The horizontal geoelectric field is calculated by applying the plane wave method (see Appendix A). The plane wave method has been shown in numerous studies to be able to accurately map the observed ground magnetic field to the geoelectric field and observed GIC [Trichtchenko and Boteler, 2004; Viljanen et al., 2006a; Wik et al., 2008]. Further, although the plane wave method assumes a one-dimensional (1-D) ground conductivity structure, the method has been shown to be 
a)

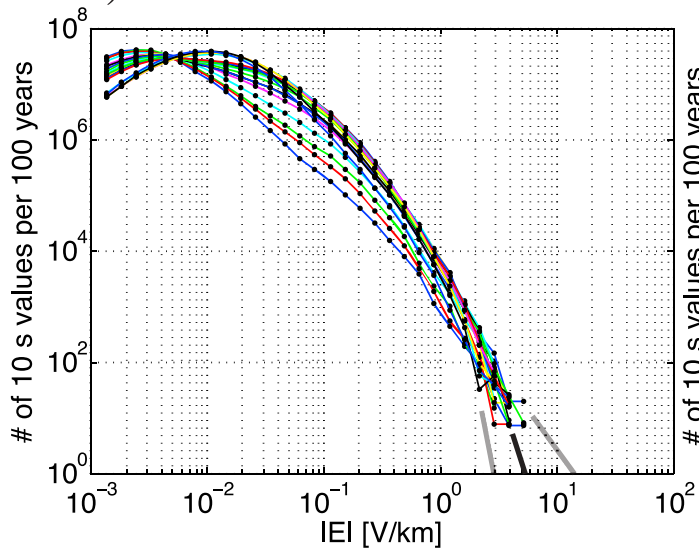

b)

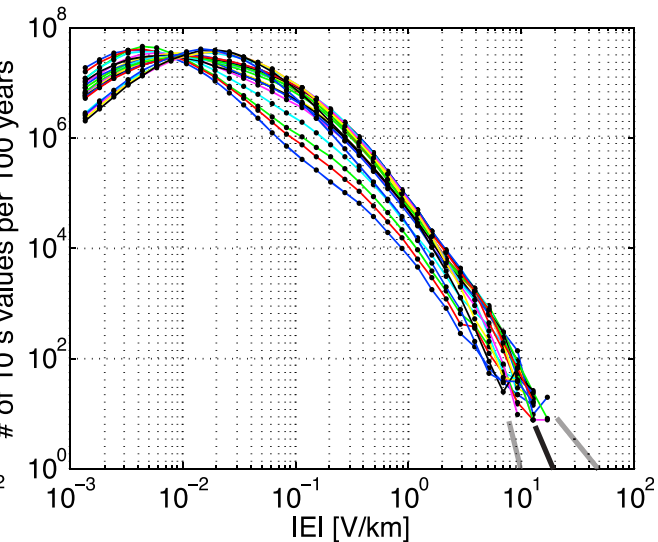

Figure 1. Statistical occurrence of the geoelectric field computed using the ground conductivity structure of (a) British Columbia, Canada (conducting) and (b) Quebec, Canada (resistive). Different curves correspond to different IMAGE stations used in the computation of the geoelectric field. The thick black lines indicate approximate visual extrapolations of the statistics to 100-year peak magnitudes. The thick grey lines indicate the reasonable lower and upper boundaries for the extrapolated values. (a) The maximum 100-year amplitude of the 10-s resolution horizontal geoelectric field is estimated to be between $3-15 \mathrm{~V} / \mathrm{km}$. (b) The maximum amplitudes are estimated to be between $10-50 \mathrm{~V} / \mathrm{km}$. The figure is a modified version of Figure 2 in Pulkkinen et al. [2008].

applicable even in highly non-1-D situations if an effective 1-D ground conductivity is used [Thomson et al., 2005; Ngwira et al., 2008; Pulkkinen et al., 2010].

[15] Pulkkinen et al. [2006] showed that while temporal averaging of the ground magnetic field from $1 \mathrm{~s}$ to $10 \mathrm{~s}$ has significant impact on peak time derivative of the ground magnetic field, the peak modeled geoelectric field amplitudes are not reduced significantly. Averaging the ground magnetic field below 10-s temporal resolution, however, was shown to impact the peak geoelectric field magnitudes [see Pulkkinen et al., 2006, Figure 9]. Consequently, 10-s data is used in the calculation of the geoelectric field. The ground conductivity structures discussed in the section below are used in the calculations. These results are then used to generate the statistical occurrence of the modeled geoelectric field at the IMAGE stations.

\subsection{The Effect of the Ground Conductivity Structure on the Extreme Amplitudes}

[16] The ground conductivity structure is one of the major factors impacting the geoelectric field magnitudes. The detailed electromagnetic response of the medium to geospace driving is dependent on the local ground conductivity structure and hence accurate calculation of the local geoelectric field and GIC requires knowledge about the local geological conditions. As the global distribution of GIC-relevant ground conductivity down to upper mantle depths (of the order of $100 \mathrm{~km}$ ) is not well known, we follow the approach used by Pulkkinen et al. [2008] and select two ground conductivity models representing realistic extreme ends of conducting (British Columbia,
Canada) and resistive (Quebec, Canada) ground structures. The resistive Quebec model, which is associated with larger geoelectric field amplitudes, will be associated with a scenario having the most extreme GIC.

[17] It is noted that, strictly speaking, one cannot apply Canadian ground models to geomagnetic observations from an entirely different geographical region as was done by Pulkkinen et al. [2008] and as is done here. However, excluding regions close to strong conductivity anomalies, to a good approximation the same magnetospheric-ionospheric source current will produce similar total magnetic field variations at regions with different ground conductivity structures. Consequently, a deviation from the strictly consistent approach in using the ground models and geomagnetic field observations is justified. For a more detailed discussion, see Pulkkinen et al. [2008].

[18] Figure 1 shows the statistical occurrence of the geoelectric field at 23 IMAGE stations for the two ground conductivity structures. Approximate visual extrapolations to 100 -year peak magnitudes are also given. As is seen from Figure 1, the peak magnitudes for different stations group quite tightly and extracting 100 -year values requires extrapolation over about one order of magnitude in occurrence rates. Further, the tails of the occurrence distributions fall off with fairly continuous slope and it is thus argued that while extraction of much rarer events may be a challenge, the presented extrapolations to 100year occurrence rates is reasonable. While one could try applying a more rigorous extreme value theory (EVT) for studying the tails of the occurrence distributions, we argue that given the approximate nature of the work at hand, 


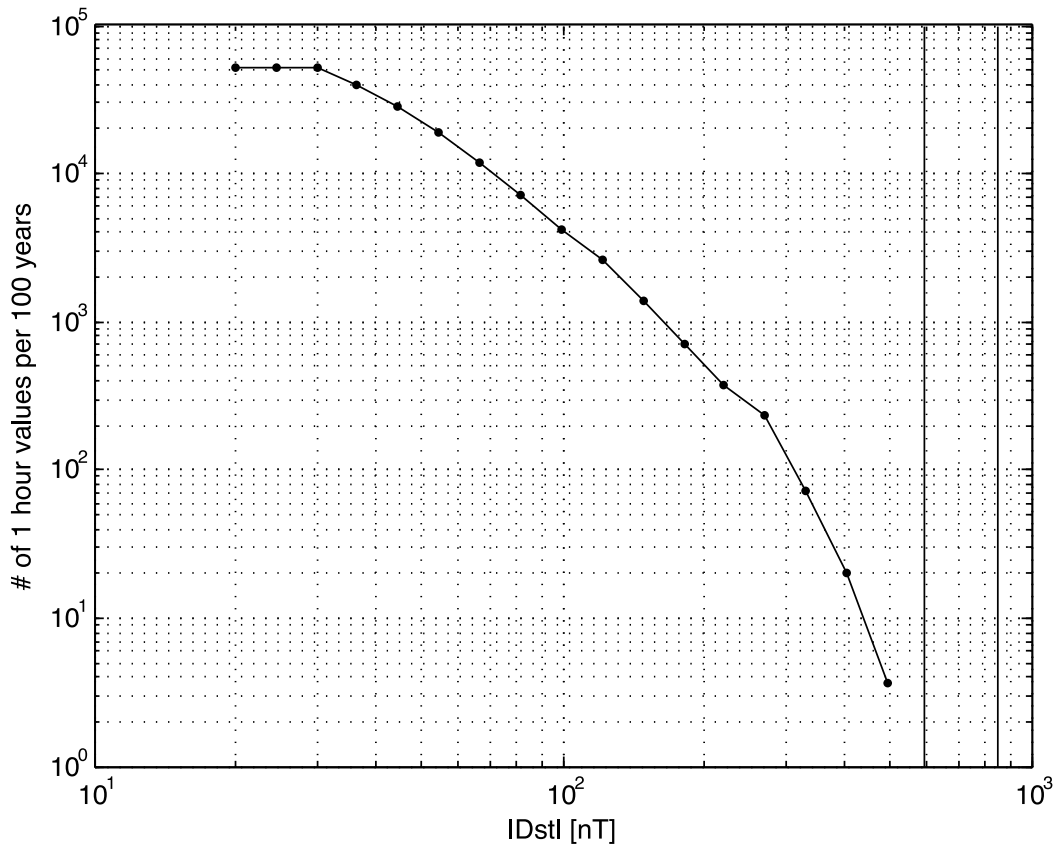

Figure 2. Statistical occurrence of hourly Dst index values between years 1957-2010. The two vertical lines indicate $|D s t|$ of $589 \mathrm{nT}$ and $850 \mathrm{nT}$.

visual extrapolation is perfectly sufficient; it is unlikely that any reasonable EVT fitting procedure would give extreme amplitudes out of the upper and lower limits indicated in Figure 1.

[19] As is seen from Figure 1, for poorly conducting (represented by Quebec ground model) high-latitude regions the maximum 100-year amplitude of the 10 -s resolution horizontal geoelectric field is estimated to be between 10 and $50 \mathrm{~V} / \mathrm{km}$. For well-conducting regions (represented by British Columbia ground model) the maximum amplitudes are about factor of 5 smaller and estimated to be between 3 and $15 \mathrm{~V} / \mathrm{km}$.

\subsection{The Effect of the Geomagnetic Latitude on the Extreme Amplitudes}

[20] Due to the location of the IMAGE magnetometer stations, the analysis in section 2.2 applies directly only to high-latitude locations between $55^{\circ}-75^{\circ}$ of geomagnetic latitude. Different magnetosphere-ionosphere source currents dominate the ground magnetic field signature at different geomagnetic latitudes. For example, at highlatitudes the magnetic signature is dominated by auroral ionospheric currents while at low-latitudes the signature is a combination of multiple sources such as ring, magnetopause, magnetotail and the equatorial electrojet currents [Ohtani et al., 2000]. Further, different magnetosphereionosphere current systems have their own spatiotemporal characteristics and consequently it is necessary to account for the geospace source variability in the generation of the extreme geoelectric field amplitudes.
[21] Unfortunately, global 10-s ground magnetic field observations are not available for extended time periods. The standard temporal resolution, for example, for the International Real-time Magnetic Observatory Network (INTERMAGNET) sites is $60 \mathrm{~s}$. Consequently, global investigations of extreme geoelectric field amplitudes are restricted to using 60-s resolution data at best, which may cut some of the peak geomagnetic field fluctuation and geoelectric field amplitudes. We assume that the relative change of the peak amplitudes as a function of geomagnetic latitude is the same for 10-s and 60-s data. While validity of this assumption cannot be verified easily for the time derivative of the magnetic field, since the geoelectric field is not as sensitive to temporal averaging [Pulkkinen et al., 2006], we argue that the available 60-s temporal resolution is sufficient for the purpose of this part of the work. Pulkkinen et al. [2006] found that the maximum modeled amplitude of the geoelectric field can be reduced by about $20 \%$ when averaging 10 -s geomagnetic data into 60 -s resolution.

[22] We studied the global behavior of the ground magnetic field and geoelectric field fluctuations for two extreme geomagnetic storm events of special significance: 13-15 March 1989 and 29-31 October 2003. The March 1989 storm caused the collapse of the Hydro Quebec highvoltage power transmission system while the October 2003 storm caused the blackout in Southern Sweden [Pulkkinen et al., 2005] and possibly problems with the South African high-voltage transmission system [Gaunt and Coetzee, 2007]. Both storms were generated by major solar coronal mass ejection events, known to be the most significant 
a)

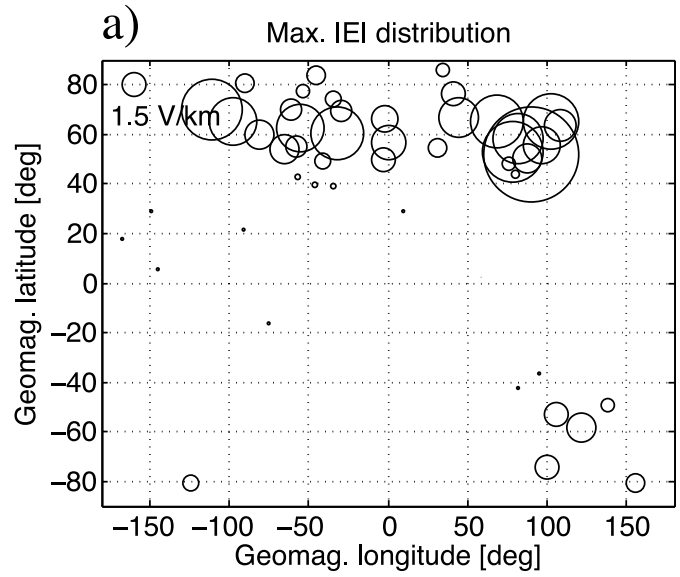

c)

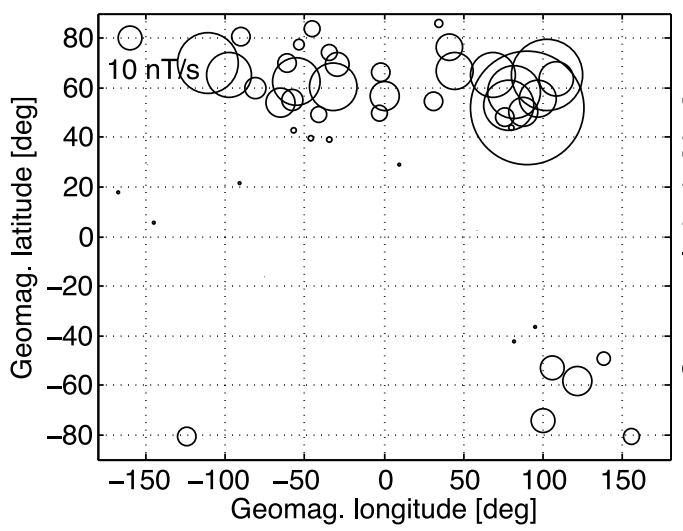

b)
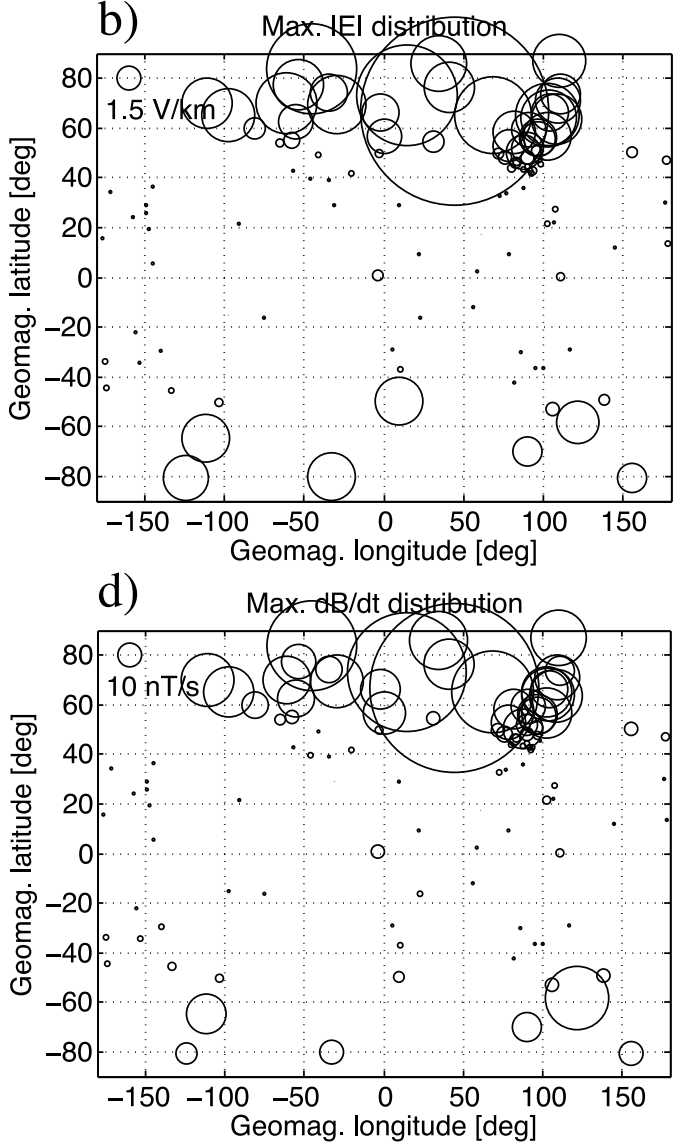

Figure 3. Spatial distributions of (top) the maximum computed geoelectric field and (bottom) the maximum time derivative of the horizontal magnetic field for $(a, c)$ the 13-15 March 1989 event and $(b, d)$ the 29-31 October 2003 event. The center of each circle indicates the location of the corresponding magnetometer station, and the radius of the circle indicates the maximum magnitudes of the physical parameters.

driver of large GIC [Borovsky and Denton, 2006; Kataoka and Pulkkinen, 2008; Huttunen et al., 2008]. The minimum Dst indices of March 1989 and October 2003 storms were -589 $\mathrm{nT}$ and $-383 \mathrm{nT}$, respectively. Using the Dst index as a measure of the storm strength, March 1989 and October 2003 storms rank between years 1957-2010 for which Dst data is available as 1st and 8th strongest, respectively. In fact, as can be seen from Figure 2 showing the statistical occurrence of hourly Dst values between 1957-2010, the peak Dst of the March 1989 storm may have been close to the 100-year amplitude. Tsubouchi and Omura [2007] used extreme value statistics to estimate that the Dst of the storm was a 60 -year event. Further, the statistics suggest that the Carrington 1859 storm peak Dst of about $-850 \mathrm{nT}$ could in fact be rarer than a 100 -year event. It is, however, noted that the transition in the slope of the distribution in Figure 2 at about $300 \mathrm{nT}$ indicates that finite size of the sample may hinder the accurate estimation of the characteristics of the tail of the distribution. Consequently, one should be careful in making interpretations about the likelihoods of the extreme Dst values.

[23] We retrieved 60-s global geomagnetic field data from INTERMAGNET (www.intermagnet.org) for the two months containing the two storm events and removed a visually determined baseline from the observations. We checked the data for obvious bad values, and stations with suspicious data were removed from the analysis. Short data gaps no longer than a few minutes were patched using linear interpolation. The Quebec ground conductivity model was then applied with the plane wave method to compute the geoelectric field at each station.

[24] Figure 3 shows the spatial distributions of the maximum computed geoelectric field and the maximum time derivative of the horizontal magnetic field taken over the 13-15 March 1989 and 29-31 October 2003 events. Figure 4 in turn shows the latitude distributions of the maximum geoelectric field, the maximum time derivative of the horizontal magnetic field and the maximum amplitude of the horizontal magnetic field. Note that in Figures 3 and 4 
a)

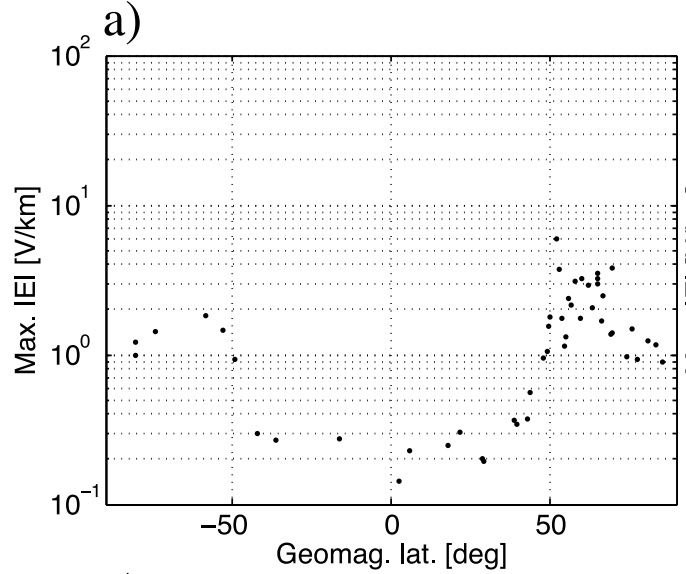

c)

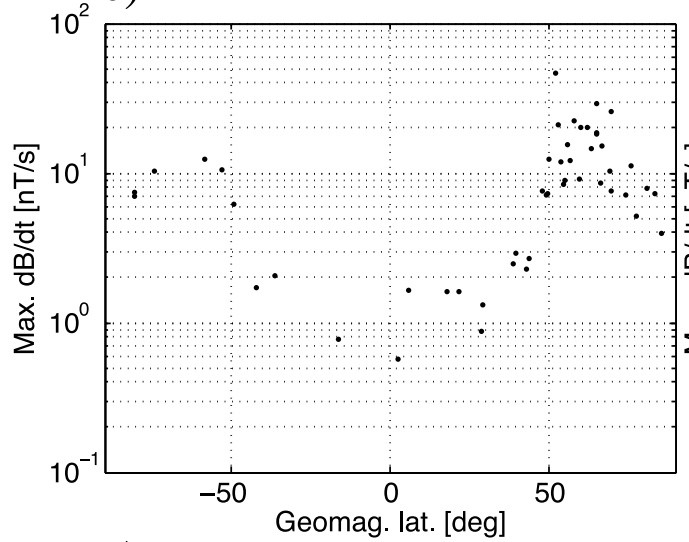

e)

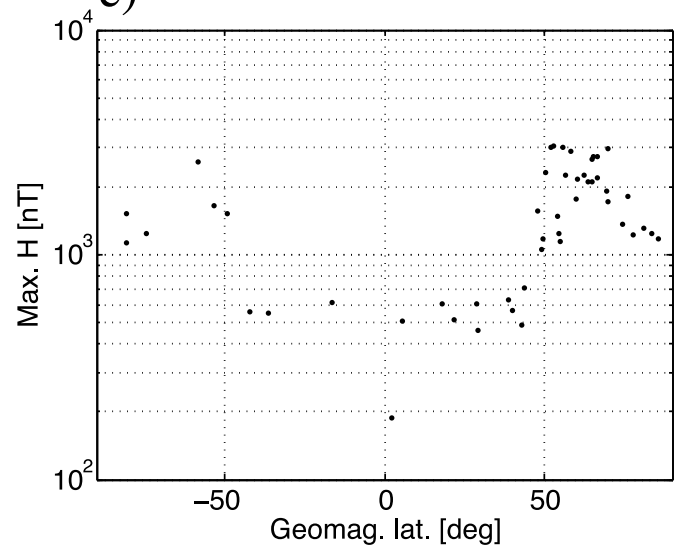

b)

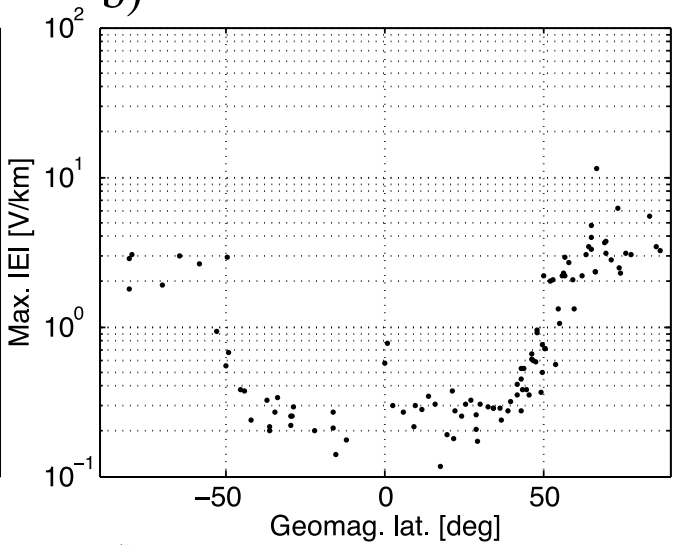

d)

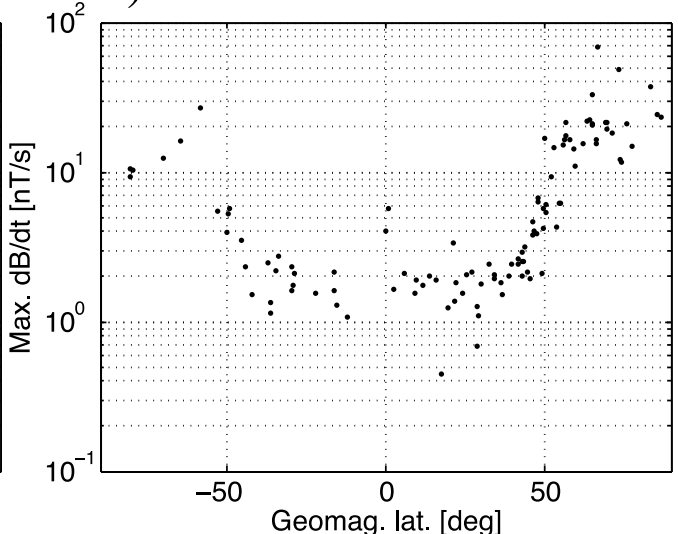

f)

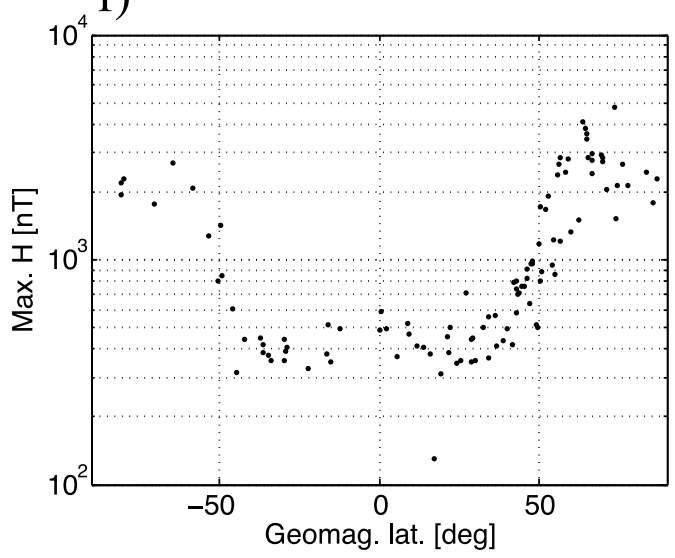

Figure 4. Geomagnetic latitude distributions of (top) the maximum computed geoelectric field, (middle) the maximum time derivative of the horizontal magnetic field, and (bottom) the maximum amplitude of the horizontal magnetic field for $(a, c, e)$ the 13-15 March 1989 event and (b, d, f) the 29-31 October 2003 events.

the maximum values at different locations can come from different individual time instants. As can be seen from Figure 3, the global coverage of the magnetometer stations especially for the October 2003 event is good. For the March 1989 event the global coverage is poorer and most observations are from the Northern Hemisphere and high-latitudes. Quite interestingly, both Figures 3 and 4 indicate a dramatic global drop in the maximum magnitudes of all three parameters approximately between $40^{\circ}$ $60^{\circ}$ of geomagnetic latitude. The maximum geoelectric field, the maximum time derivative of the horizontal magnetic field and the maximum amplitude of the 


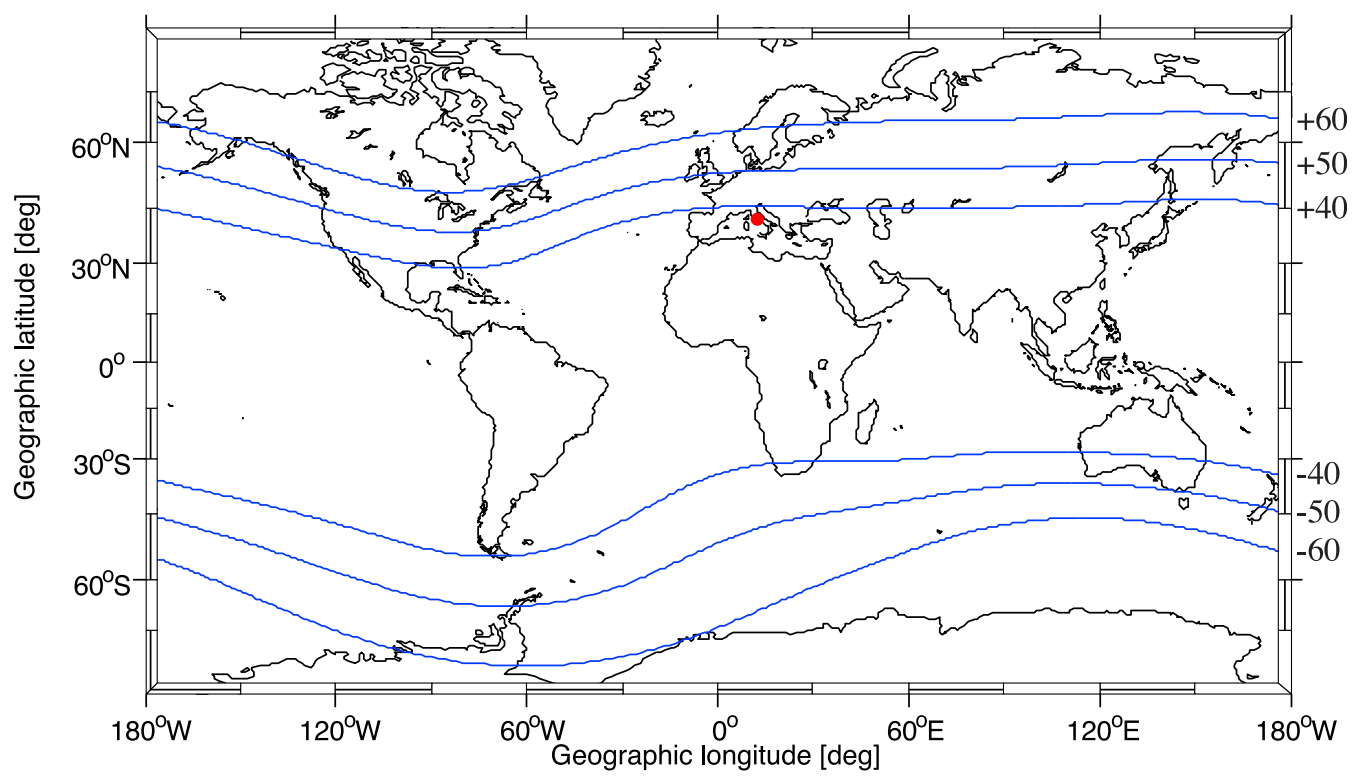

Figure 5. This geographic map displays $-60^{\circ},-50^{\circ},-40^{\circ}, 40^{\circ}, 50^{\circ}$ and $60^{\circ}$ of geomagnetic latitude contours (blue lines). The red dot indicates the location of Rome, Italy (as discussed further in the text). Altitude adjusted corrected geomagnetic coordinate coefficients for year 2000 were used in the generation of the contours. Coordinates were calculated for zero altitude.

horizontal magnetic field all experience approximately an order of magnitude drop across the threshold at about $50^{\circ}$ of geomagnetic latitude. Furthermore, the same drop is observed both in the Northern and Southern hemispheres. We also note that there is a tendency for both events to have slightly larger field amplitudes at the Northern Hemisphere for all parameters in Figures 3 and 4. More detailed study of the reason for this new observation, however, is out of scope of the work presented in this paper.

[25] Figures $3 b, 3 d, 4 b$, and $4 d$ show confined enhancement of maximum computed geoelectric field and the maximum time derivative of the horizontal magnetic field for two stations at about the magnetic equator. The maximum values occurred between 08:00-12:00 magnetic local time (not shown), which indicates that the enhancement may be associated with the equatorial electrojet that is a localized band of ionospheric current between about $-5^{\circ}$ to $5^{\circ}$ of geomagnetic latitude [Lühr et al., 2004]. To our knowledge, this is the first time that the equatorial electrojet has been shown to be capable of significantly enhancing GIC. Again, however, a more detailed study is beyond the scope of this paper.

[26] One of the interesting features in Figures 3 and 4 is the implied universality of the threshold at about $50^{\circ}$ of geomagnetic latitude. In terms of Dst index the March 1989 storm was significantly stronger than the October 2003 storm. Consequently, one could expect that, for example, the auroral boundaries would have penetrated to significantly lower latitudes during the March 1989 event. However, the drop of the maximum amplitudes occurs for both storm events at approximately $40^{\circ}-60^{\circ}$ of geomagnetic latitude. Clearly usage of global geomagnetic data for extended time periods is required to study and confirm the location and dynamics of the threshold geomagnetic latitude more definitively. However, Thomson et al. [2011] used 28 years of 60-s geomagnetic data from Europe and found a similar threshold at about $55^{\circ}$ of geomagnetic latitude for the time derivative of the horizontal magnetic field. We thus conjecture that the threshold at about $50^{\circ}-55^{\circ}$ of geomagnetic latitude is a universal feature of most major or extreme geomagnetic storms (for geographical reference, see Figure 5).

[27] As is seen in Figure 4, the threshold latitude for the maximum geoelectric field and the maximum time derivative of the horizontal magnetic field is reflected in the maximum amplitude of the horizontal magnetic field. This is an indication that the most extreme magnetic field fluctuations and geoelectric field magnitudes are associated with the auroral current system that is known to be responsible for the largest perturbations of the ground magnetic field. For example, while magnetospheric ring current can generate horizontal magnetic field perturbations of the order of hundreds of nT, auroral currents regularly generate perturbations of the order of thousands of $\mathrm{nT}$. Even in extreme cases as for the Carrington event the low-latitude maximum perturbation of about $1600 \mathrm{nT}$ [Tsurutani et al., 2003] is smaller than the typical storm-time perturbations at auroral regions. Consequently, the question about the location of the threshold geomagnetic latitude can be cast also in terms of the maximum possible expansion of the auroral current system.

[28] Possibly the strongest geomagnetic storm in recorded history is the Carrington event of 1-2 September 1859 
Table 1. The Approximate Maximum Equatorward Auroral Boundary Locations of Selected Extreme Geomagnetic Storm Events $^{\text {a }}$

\begin{tabular}{lc}
\hline \multicolumn{1}{c}{ Event Date } & Location in Geomagnetic Latitude \\
\hline 28-29 August 1859 & $48^{\circ \mathrm{b}}$ \\
1-2 September 1859 & $41^{\circ \mathrm{b}}$ \\
14-15 May 1921 & $40^{\circ \mathrm{b}}$ \\
13-15 March 1989 & $40^{\circ \mathrm{c}}$ \\
\hline
\end{tabular}

${ }^{\mathrm{a}}$ This table is based on data presented by Allen et al. [1989], Yokoyama et al. [1998], and Silverman and Cliver [2001].

${ }^{\mathrm{b}}$ Maximum equatorward extent of the overhead visual aurora.

${ }^{c}$ Maximum equatorward extent in DE-1 imagery and electron precipitation boundary from the Defense Meteorological Satellite Program.

[Tsurutani et al., 2003; Siscoe et al., 2006]. The minimum estimated Dst index of the storm was $-850 \mathrm{nT}$ and there were (poleward horizon) auroral sightings from as low as $23^{\circ}$ of geomagnetic latitude. However, from the viewpoint of the analysis in this paper, perhaps the most significant observation during the event comes from Rome, Italy (see Figure 5). More specifically, a perturbation of the order of $3000 \mathrm{nT}$ was observed using a bifilar magnetometer that indicates the relative changes in the horizontal magnetic field strength [Loomis, 1860]. The geomagnetic latitude of Rome, Italy, is about $36^{\circ}$ and comparing this to Figures $4 \mathrm{e}$ and $4 \mathrm{f}$, the observation indicates that the maximum expansion of the auroral current system may have been about $20^{\circ}$ more southward than during the March 1989 or October 2003 storms. Although this may sound somewhat fantastic and the single data point did not arise from modern scientific instrumentation, one cannot simply disregard the Rome observation. It may thus be possible that during the most extreme geomagnetic storms the auroral current system and the accompanying extreme geoelectric fields and GIC can penetrate significantly below the threshold of about $50^{\circ}$ of geomagnetic latitude. Unfortunately, due to the poor spatial coverage, low temporal sampling rates and off-scale magnitudes, magnetic recordings of the Carrington event do not allow for more detailed analysis of the global geoelectric field and GIC characteristics [Nevanlinna, 2006; Boteler, 2006; Nevanlinna, 2008]. At sub-auroral latitudes in Finland and Russia, the greatest measured hourly point deviations in the horizontal field during the event were about $1000 \mathrm{nT}$ [Nevanlinna, 2008].

[29] We suggest that the threshold geomagnetic latitude can be investigated also by means of observations of the low-latitude boundary for auroral emissions (indicative of the general location of the auroral region). Records of auroral sightings are available for certain historical storms and these enable approximate reconstructions of the auroral region morphology during the corresponding storms. For example, Silverman and Cliver [2001], Cliver and Svalgaard [2004], and Silverman [2006] provide auroral data collected from numerous catalogues and earlier studies. Most importantly, Silverman and Cliver [2001] provide the maximum equatorward extent of the visual aurora for three historical extreme geomagnetic storms: 28-29 August 1859, 1-2 September 1859, and 14-15 May 1921. While the 28-29 August 1859 event was part of the AugustSeptember 1859 extreme storm sequence, analysis by Kappenman [2006] indicates that minimum Dst index of the 14-15 May 1921 event may have been comparable to that of the Carrington event. Silverman and Cliver [2001] provide auroral boundary locations both for the overhead auroras and auroras observed in the poleward horizon. However, only the overhead auroral sightings provide unambiguous determination of the auroral boundary location and thus auroras observed in the poleward horizon are not discussed further here.

[30] Table 1 shows the approximate low-latitude auroral boundary locations for the four extreme storm events. The boundary location for the 13-15 March 1989 was determined by visual inspection of Dynamics Explorer 1 (DE-1) ultraviolet auroral emission imaging data for 14 March 1989, 01:51 UT [in Allen et al., 1989]. The visually determined boundary at about $40^{\circ}$ of geomagnetic latitude is in very good agreement with the electron precipitation boundary determined from low-Earth orbit Defense Meteorological Satellite Program (DMSP) satellite data presented by Yokoyama et al. [1998].

[31] The striking feature of the data in Table 1 is that the boundary is confined to approximately $40^{\circ}$ of geomagnetic latitude or greater for all four events. This is also the approximate low-latitude boundary for the transition from low-latitude maximum field magnitudes to high-latitude maximum field magnitudes seen in Figure 4. As auroral emissions are congruent with the auroral ionospheric current fluctuations, low-latitude auroral boundary locations for extreme storm events in Table 1, along with "calibration" to modern magnetic field observations via the 13-15 March 1989 event in Figure 4, provide further indication of possible generality of the threshold at about $50^{\circ}$ of geomagnetic latitude. Although their results were based on data only for year 1998, Ahn et al. [2005] also concluded that the lowest possible latitude of the center of the ionospheric westward electrojet seems to be at around $60^{\circ}$ of geomagnetic latitude, which is consistent with Figures 3 and 4. Ahn et al. [2005] also showed that the lowlatitude boundary of auroral emissions tends to locate equatorward of the westward electrojet, which is also consistent with our findings above.

[32] It is noted that the Rome observation of geomagnetic field perturbation of the order of $3000 \mathrm{nT}$ during the Carrington event contradicts the idea of the threshold geomagnetic latitude at about $50^{\circ}$ given the $40^{\circ}$ of geomagnetic latitude boundary for the event in Table 1 . The possible explanations for the discrepancy are (1) lowlatitude boundary of the auroral emissions is not always congruent with the low-latitude boundary of the auroral ionospheric currents, (2) the actual low-latitude boundary of the overhead auroral emissions was at the time of the Rome observation lower than $40^{\circ}$ of geomagnetic latitude but was not captured by any of the historical records and (3) the Rome magnetic field observation was erroneous. 
While the first possibility seems especially unlikely, further work is needed to find the most plausible explanation for the discrepancy.

[33] In conclusion, given the very similar properties of the March 1989 and October 2003 storms shown in Figures 3 and 4 along with statistics given by Thomson et al. [2011], we are inclined to maintain our conjecture that the threshold at about $50^{\circ}-55^{\circ}$ of geomagnetic latitude holds for most major and extreme geomagnetic stormsand possibly for 100-year events as well. In terms of extreme event scenarios and scaling, this means that the extreme geoelectric field amplitudes drop by about a factor of 10 across the region from $60^{\circ}-40^{\circ}$ of geomagnetic latitude (see Figure 5). However, we emphasize again that more extreme geomagnetic storm data is required to reach any definite conclusions regarding the 100 -year location of the threshold geomagnetic latitude.

\subsection{Temporal Scales of the Extreme Events}

[34] Many different types of dynamical processes in the solar wind-magnetosphere-ionosphere system are capable of generating large GIC. For example, auroral substorms, geomagnetic pulsations, sudden impulses and enhancements of magnetospheric convection-related auroral electrojets are known to drive GIC [Anderson et al., 1974; Kappenman, 2003; Pulkkinen et al., 2003; Viljanen et al., 2006b]. All of these processes have their distinct spectral characteristics and thus no single simple synthetic temporal profile is capable of capturing the full temporal variability observed during extreme storms [Pulkkinen and Kataoka, 2006; Kataoka and Pulkkinen, 2008]. Consequently, we chose to use an actual geomagnetic storm event to provide a representative temporal profile for the generated scenarios. We selected 10-s geomagnetic field observations from Nurmijärvi Geophysical Observatory, Finland and Memanbetsu Geophysical Observatory, Japan for the period of 29-31 October 2003 to provide the temporal profiles for the scenarios. The Nurmijärvi Geophysical Observatory is located approximately at $57^{\circ}$ of geomagnetic latitude and was thus within the region experiencing the most extreme magnetic field fluctuations above the threshold geomagnetic latitude during the storm. Memanbetsu Geophysical Observatory in turn is located approximately at $35^{\circ}$ of geomagnetic latitude and was thus within the region experiencing the magnetic field fluctuations below the threshold geomagnetic latitude during the storm. We also confirmed that the selected representative storm profiles include signatures of auroral substorms, geomagnetic pulsations, sudden impulses and enhancements of magnetospheric convection-related auroral electrojets. The selected magnetometer stations and the geomagnetic storm event thus provide a good representation of the ground electromagnetic field fluctuations during major geomagnetic storms.

[35] Geomagnetic field observations from Nurmijärvi and Memanbetsu Geophysical Observatories for 29-31
October 2003 were applied with the Quebec ground conductivity model and the plane wave method to map the geomagnetic field into the horizontal geoelectric field. The obtained geoelectric field time series was then normalized so that the maximum amplitude of the signal is exactly 1 (Figure 6), i.e.,

$$
\max \left(\sqrt{E_{x}^{2}+E_{y}^{2}}\right)=1,
$$

where $E_{x}$ and $E_{y}$ are the normalized horizontal geoelectric field components, and the maximum is taken over the storm event. The normalized horizontal geoelectric field is the signal that is used to scale to different maximum 100year amplitude scenarios as a function of ground conductivity structures and geomagnetic latitudes.

\subsection{Spatial Scales of the Extreme Events}

[36] In the challenge of generating 100-year geoelectric field and GIC scenarios, characterizing the spatial scales of the extreme events may be the most difficult task. As was discussed above, many different types of processes in the solar wind-magnetosphere-ionosphere system drive large GIC and each of these processes have their characteristic temporal and spatial scales. The global spatial scales of the geoelectric field associated with these processes are, in general, not well known. High-latitude (auroral) magnetic field and geoelectric field fluctuations tend to be poorly correlated over distances greater than $100 \mathrm{~km}$ [Pulkkinen, 2007 , and references therein]. Since the extreme geomagnetic field and geoelectric field fluctuations associated with enhancements of the auroral current system can be global, so these two aspects give rise to a twofold view: while large or extreme geoelectric field magnitudes can be experienced across the globe in the region covered by the high-latitude auroral current system, the spatial correlation lengths associated with the field fluctuations can be short. Further complications are caused by the horizontal variations in the ground conductivity structure. The electromagnetic response to geospace driving is a strong function of the ground conductivity structure and steep horizontal conductivity gradients can generate steep fluctuations in the spatial geoelectric field structure [Thomson et al., 2005].

[37] Since we have no means of generating a global geoelectric field structure that would represent with any reasonable accuracy the true spatial scales (and spatial correlations) of the extreme fields, we are constrained to represent fields in regional scales. Consequently, we will assume that the geoelectric field is uniform over spatial scales of the order of $100-1000 \mathrm{~km}$. In other words, the geoelectric field of the extreme storm scenarios has the same instantaneous direction and magnitude throughout the region of interest. The spatial uniformity amounts to assuming that there are no significant horizontal variations in the ground conductivity structure or in the spatial structure of the source field fluctuations. It is emphasized 
a)
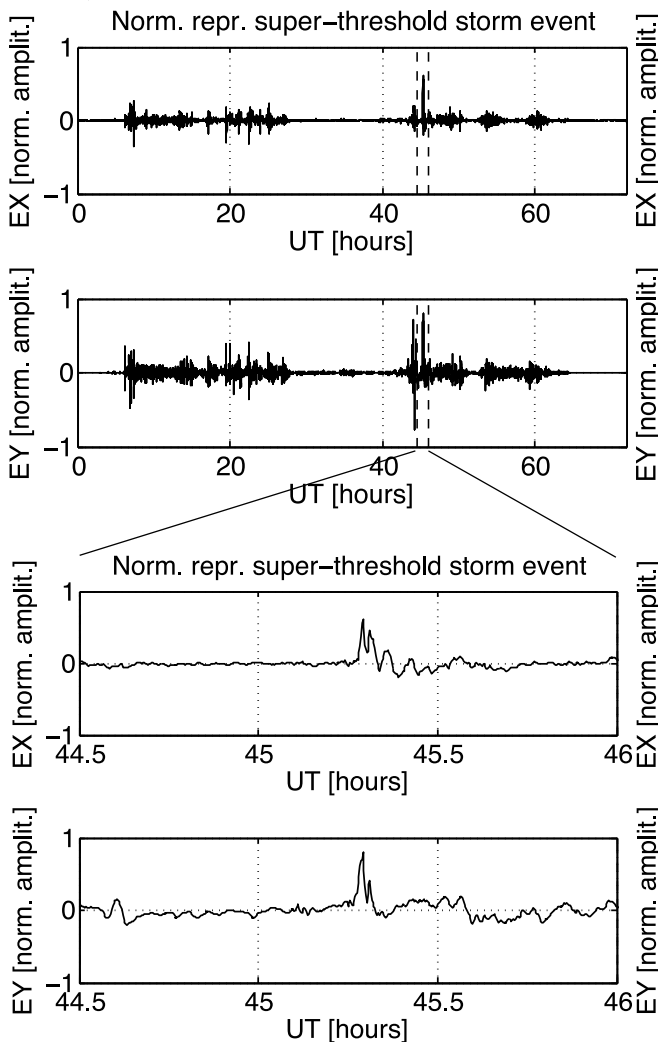

b)
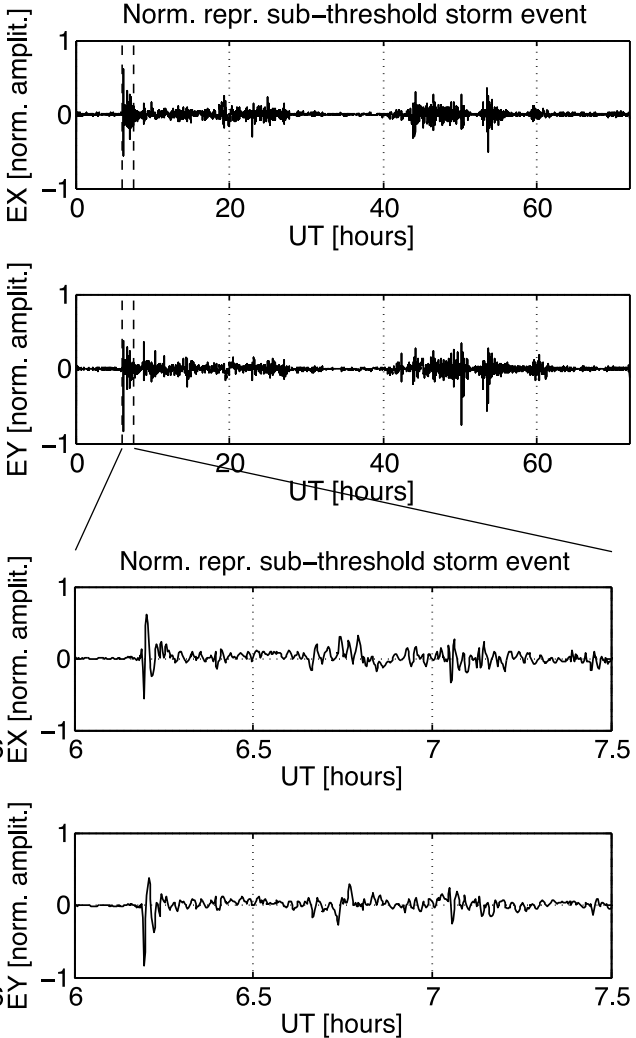

Figure 6. Normalized representative horizontal geoelectric field components ( $X$ indicates geographic north, $Y$ indicates geographic east) for the full storm event and $1.5 \mathrm{~h}$-long subsections containing the maximum field magnitude for (a) super-threshold geomagnetic latitude locations represented by Nurmijärvi Geophysical Observatory and (b) sub-threshold geomagnetic latitude locations represented by Memanbetsu Geophysical Observatory. The time is hours from 29 October 2003, 00:00 UT. The maximum geoelectric field magnitude at Nurmijärvi was caused by an auroral substorm, while the maximum field magnitude at Memanbetsu was caused by a sudden impulse. See the text for details.

that while these assumptions may be reasonable for the purpose of the extreme scenarios on short regional scales, they will break and should not be used on global scales.

\section{Summary of the Extreme Geoelectric Field Scenarios}

[38] Summarizing the findings in section 2, the four key factors introduced in section 1 are addressed in the extreme geoelectric field scenarios in the following ways:

[39] - The effect of the ground conductivity structure on the extreme geoelectric field amplitudes: Two ground conductivity models representing realistic extreme ends of conducting and resistive grounds were applied with the IMAGE magnetometer data and the plane wave method. The results were used to estimate 100 -year amplitudes of the 10-s resolution horizontal geoelectric field at high latitudes. The resistive ground model is associated with geoelectric field amplitudes approximately five times larger than those seen with the conducting ground model.

[40] - The effect of the geomagnetic latitude on the extreme geoelectric field amplitudes: We identified a threshold geomagnetic latitude across which the maximum geoelectric field amplitudes experience approximately an order of magnitude decrease toward the equator.

[41] - Temporal scales of the extreme geoelectric fields: Representative time series from selected magnetometer stations for a major storm event were used to provide realistic temporal profiles. Stations above and below the identified threshold geomagnetic latitude were used.

[42] - Spatial scales of the extreme geoelectric fields: We assume spatially uniform geoelectric field structure in regional scales.

[43] Figure 7 summarizes the four 100-year extreme geoelectric field scenarios. In Figures $7 \mathrm{a}$ and $7 \mathrm{~b}$ the normalized geoelectric field in Figure 6a was scaled using the 
a)
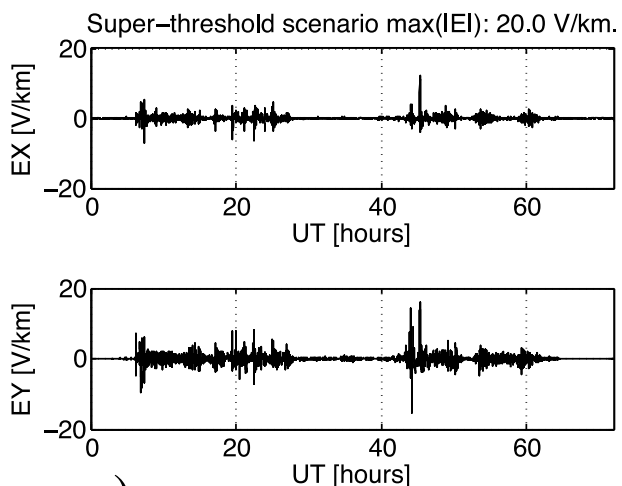

c)
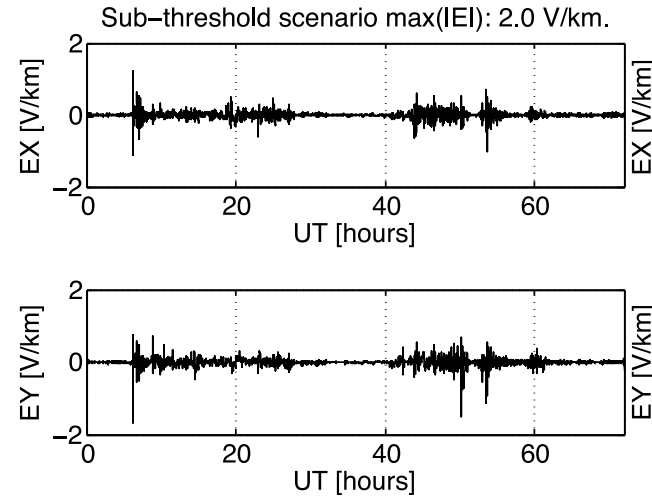

b)
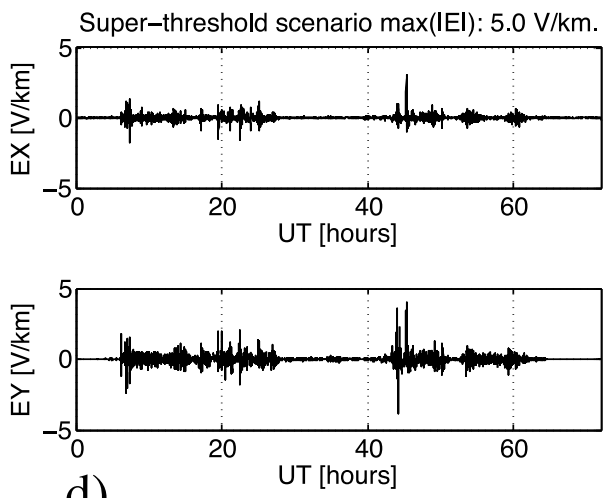

d)
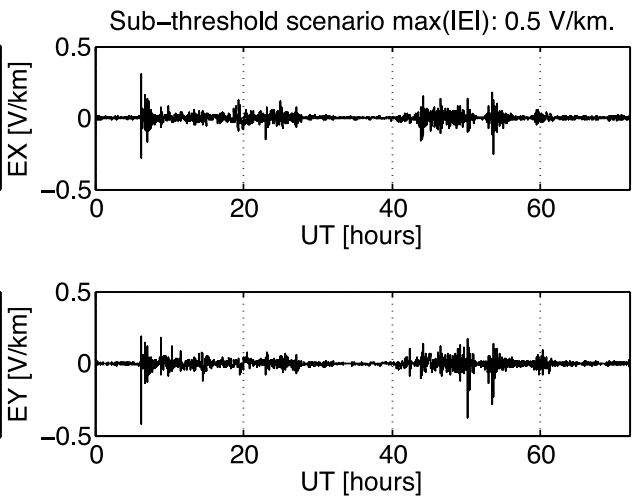

Figure 7. Illustration of extreme horizontal geoelectric field scenarios ( $X$ indicates geographic north, $Y$ indicates geographic east). (a) Scenario for resistive ground structures for locations above the threshold geomagnetic latitude. The maximum geoelectric field amplitude is $20 \mathrm{~V} / \mathrm{km}$. (b) Scenario for conductive ground structures for locations above the threshold geomagnetic latitude. The maximum geoelectric field amplitude is $5 \mathrm{~V} / \mathrm{km}$. (c) Scenario for resistive ground structures for locations below the threshold geomagnetic latitude. The maximum geoelectric field amplitude is $2 \mathrm{~V} / \mathrm{km}$. (d) Scenario for conductive ground structures for locations below the threshold geomagnetic latitude. The maximum geoelectric field amplitude is $0.5 \mathrm{~V} / \mathrm{km}$. Note that the vertical scales are different in different panels.

maximum amplitudes of $20 \mathrm{~V} / \mathrm{km}$ and $5 \mathrm{~V} / \mathrm{km}$ obtained from the high-latitude statistics in Figure 1. In Figures 7c and $7 \mathrm{~d}$ the normalized geoelectric field in Figure $6 \mathrm{~b}$ was scaled by order of magnitude smaller maximum field strengths for sub-threshold geomagnetic latitudes. The threshold geomagnetic latitude can be set at $50^{\circ}$ or, for more conservative estimates, at $40^{\circ}$ of geomagnetic latitude. The geoelectric field is assumed spatially uniform in regional scales for all scenarios in Figure 7.

\section{Mapping Geoelectric Field Scenario to Geomagnetically Induced Currents}

[44] Mapping the extreme geoelectric field scenarios into GIC is a highly system-dependent operation. The response of the conductor system is dependent on the electrical characteristics and topology of the system and consequently it is generally speaking not feasible to provide any "prototype" configuration that could be applied to a variety of different situations. In other words, one needs to have additional engineering information available about the characteristics of the conductor system of interest prior to mapping the geoelectric field into GIC.

[45] Once the engineering information about the system has been acquired, there are two fairly straightforward means to carry out the mapping. First, if computation of GIC distribution throughout the (regional) system is needed, one can apply techniques used by Lehtinen and Pirjola [1985] for discretely grounded systems, such as high-voltage transmission systems, and so-called distributed source transmission line (DSTL) theory for continuously grounded systems, such as buried oil and gas pipelines [Boteler, 1997].

[46] To demonstrate the application of the extreme geoelectric field scenarios in computing GIC distribution throughout a high-voltage power transmission system, we considered Dominion Virginia Power's grid model, shown 


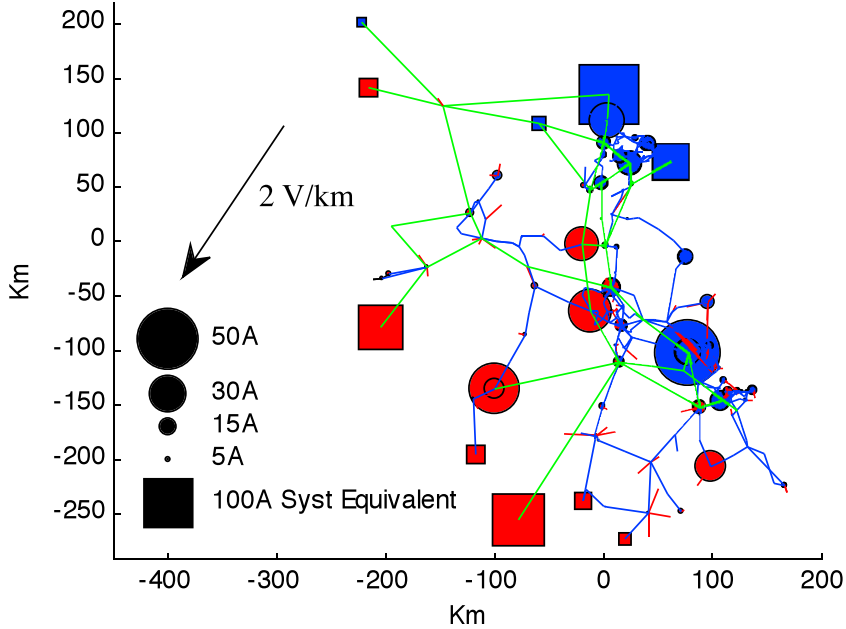

Figure 8. Modeled geomagnetically induced current (GIC) distribution in Dominion Virginia Power's highvoltage transmission system for hour 6.17 in the scenario shown in Figure 7c. Green, blue and red lines indicate $500 \mathrm{kV}, 230 \mathrm{kV}$ and $115 \mathrm{kV}$ transmission lines, respectively. The black arrow indicates the direction and magnitude of the horizontal geoelectric field, and blue and red circles indicate the magnitude of GIC flowing from the ground to the grid and from the grid to the ground, respectively. For auto-transformers, an effective GIC value is used [Albertson et al., 1981]. System equivalents-i.e., inter-ties to other systemsare represented by squares.

in Figure 8. The model is built based on a DC-mapping of Dominion's high-voltage transmission network. Typically, due to the scales associated with the GIC phenomenon, DC models should include the $500 \mathrm{kV}$ and the $230 \mathrm{kV}$ networks; a few key $115 \mathrm{kV}$ transmission lines are also considered in this particular model. Since the geoelectric field is assumed spatially uniform across the system, transmission lines between the transformers can be represented as straight lines. At each system inter-tie, a system equivalent is approximated as a low resistance to ground $(0.1 \Omega)$.

[47] The calculation of GIC flows in Dominion's highvoltage transmission system is performed by using the matrix formulation derived by Lehtinen and Pirjola [1985]. In general, GIC flows are a function of system topology, line resistances, geospatial orientation, transformer type and winding resistance, grounding resistance, series line compensation, and of course, geoelectric field. Figure 8 shows a snapshot of GIC flows at each transformer, on a per-phase basis, caused by the maximum amplitude of the geoelectric field in the scenario shown in Figure 7c. The selection of the storm scenario is based on Dominion's geomagnetic latitude (below the threshold latitude) and ground conductivity (resistive ground). To demonstrate that the approach provides a time series of GIC throughout the system over the entire storm scenario, Figure 9 shows times series of modeled GIC flow in one of Dominion's transformers. Note that the approach provides corresponding time series for any location in the highvoltage transmission system.

[48] To put the results for Dominion Virginia Power in context, Figures 10 and 11 show corresponding results for GIC flowing in a simplified model of the U.K. high-voltage system. As described by Thomson et al. [2005] and Turnbull [2011], the U.K. system (here a model for year 2008) is a highly complex system of $26,670 \mathrm{~km}$ of $132 \mathrm{kV}, 275 \mathrm{kV}$ and $410 \mathrm{kV}$ line connecting around 680 transformer substations. Modeling considerations for the U.K. system are discussed further by Thomson et al. [2005] and Turnbull [2011] as well as by Beamish et al. [2002].

[49] The model used here is of the $275 \mathrm{kV}$ and $410 \mathrm{kV}$ part of the U.K. grid. One simplification we have adopted is that all lines share the same resistance. Similarly, all earth nodes have a common resistance, different from that of the line resistances. One line is also assumed to connect neighboring nodes in the network. In reality nodes may be connected by single or double circuits and each node may contain different number of transformers, each carrying three phases of current. Thus estimated per-node GIC is subdivided, in practice, between transformers and phases, depending on the relative resistances of each component.

[50] The U.K. differs from Virginia in that it is ACisolated from neighboring national grids, though there exists DC interconnections to Europe and Ireland. Also in contrast to Virginia, U.K. is an island. In this study, however, we have disregarded the influence of the surrounding conducting seawater, which serves to enhance geoelectric fields near the coasts, and may enhance measured GIC at nodes of the network at coastal sites. That is, we assume that the extreme geoelectric field applies across a region bounding the U.K. mainland. In Figures 10 and 11 we show results where we have split the United Kingdom north and south at approximately $50^{\circ}$ of geomagnetic latitude. North of the $50^{\circ}$ boundary we assume the scenario

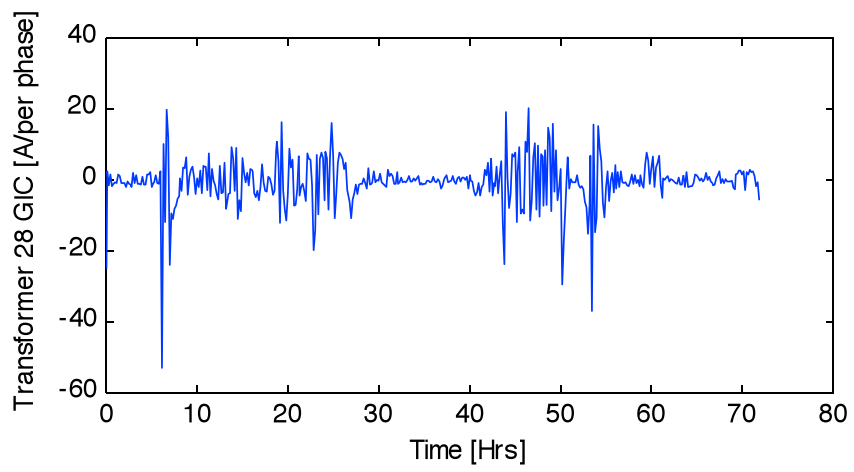

Figure 9. Time series of the modeled GIC in one of Dominion Virginia Power's high-voltage transmission system transformers for the extreme geoelectric field scenario in Figure 7c. The configuration of the transmission system is shown in Figure 8. Only maximum amplitude GIC taken over 10-min windows are shown. 


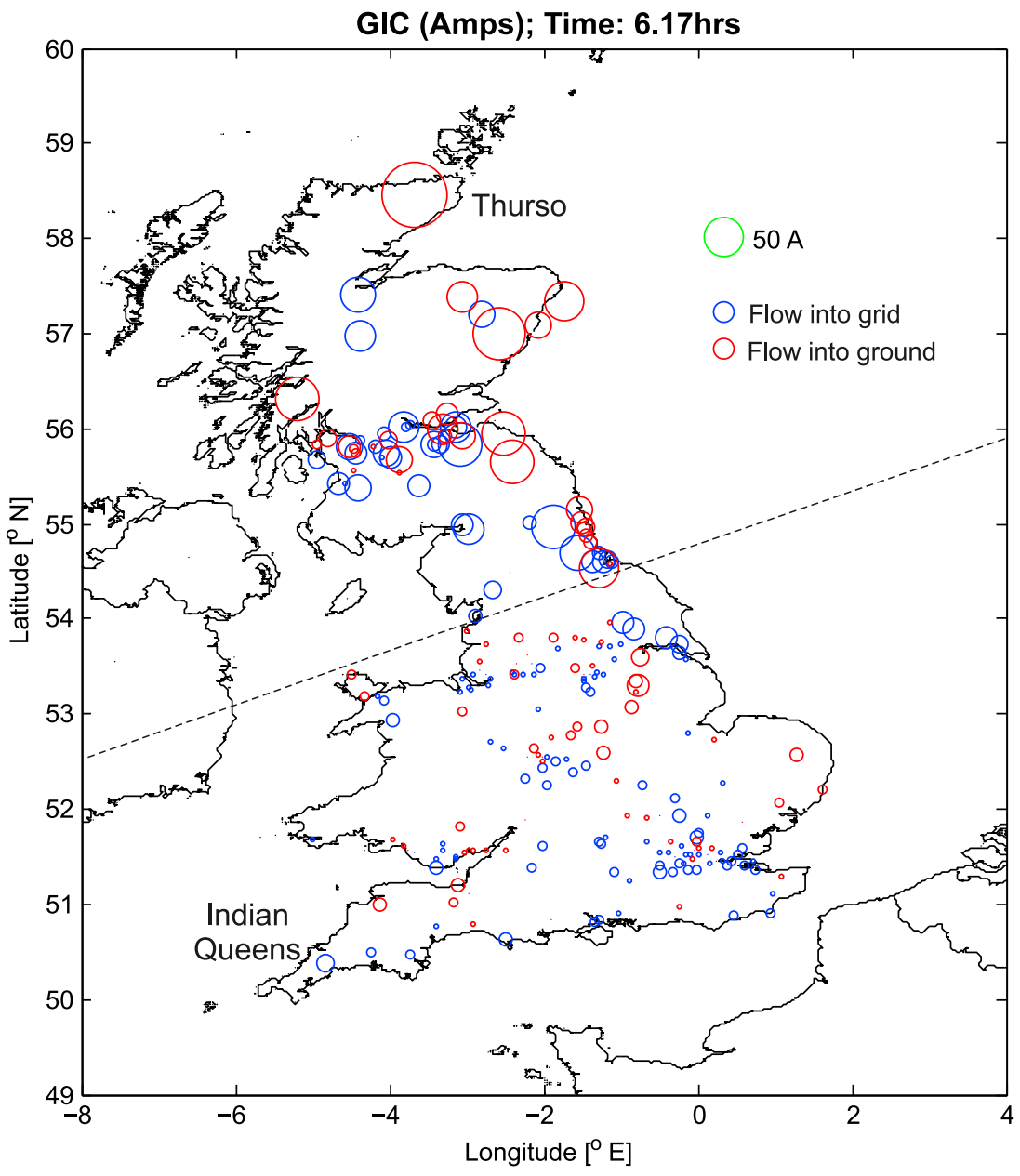

Figure 10. A snapshot of the magnitude (circle size) and direction of flow (red denotes current flowing to Earth; blue into the grid) for GIC per node in a simplified model of the U.K. high-voltage power transmission system. The scenario used is a combination of the scenarios shown in Figures $7 \mathrm{a}$ (resistive ground north of $50^{\circ}$ geomagnetic latitude) and $7 \mathrm{~d}$ (conducting ground south of $50^{\circ}$ geomagnetic latitude), with the delineation between regions approximated by the dashed line. Figure 10 shows a modeled GIC peak at time $6.17 \mathrm{~h}$.

shown in Figure 7a, and south of the boundary we assume the scenario shown in Figure 7d. This more complex scenario, i.e., with different source fields and crustal structure across the country, is closer to the situation in the "real" U.K. [Beamish et al., 2002; Thomson et al., 2011].

[51] In Figure 10 we see that there is a complicated distribution of GIC in the grid for the used scenario: for example GIC does not increase uniformly with latitude, nor does the sign of GIC change consistently from east to west. This observation agrees with distributions of GIC obtained for real events [e.g., Thomson et al., 2005]. As is seen from Figure 11, GIC at Thurso and Indian Queens are quite different from the values obtained for the Dominion Virginia Power transformer site shown in Figure 9. Given the approximate nature of the scenarios and the grid model, Figures 10 and 11 may be best seen as being indicative of the U.K. grid's geographical response to extreme conditions in terms of relative severity and location of the "hot spots" rather than as providing accurate predictions of the magnitude of GIC at each node.

[52] Finally, in contrast to a more detailed modeling demonstrated above for the Dominion Virginia Power and U.K. grids, if only local GIC flowing through individual node of power transmission system is needed, one can apply the simple linear relation

$$
\text { GIC }=a E_{x}+b E_{y}
$$

where $\left(E_{x}, E_{y}\right)$ are the horizontal components of the geoelectric field and $(a, b)$ the system parameters. The parameters $(a, b)$ depend on the topology and electrical characteristics of the conductor system under investigation and can be derived for individual locations by using information about the full conductor system [Pulkkinen 

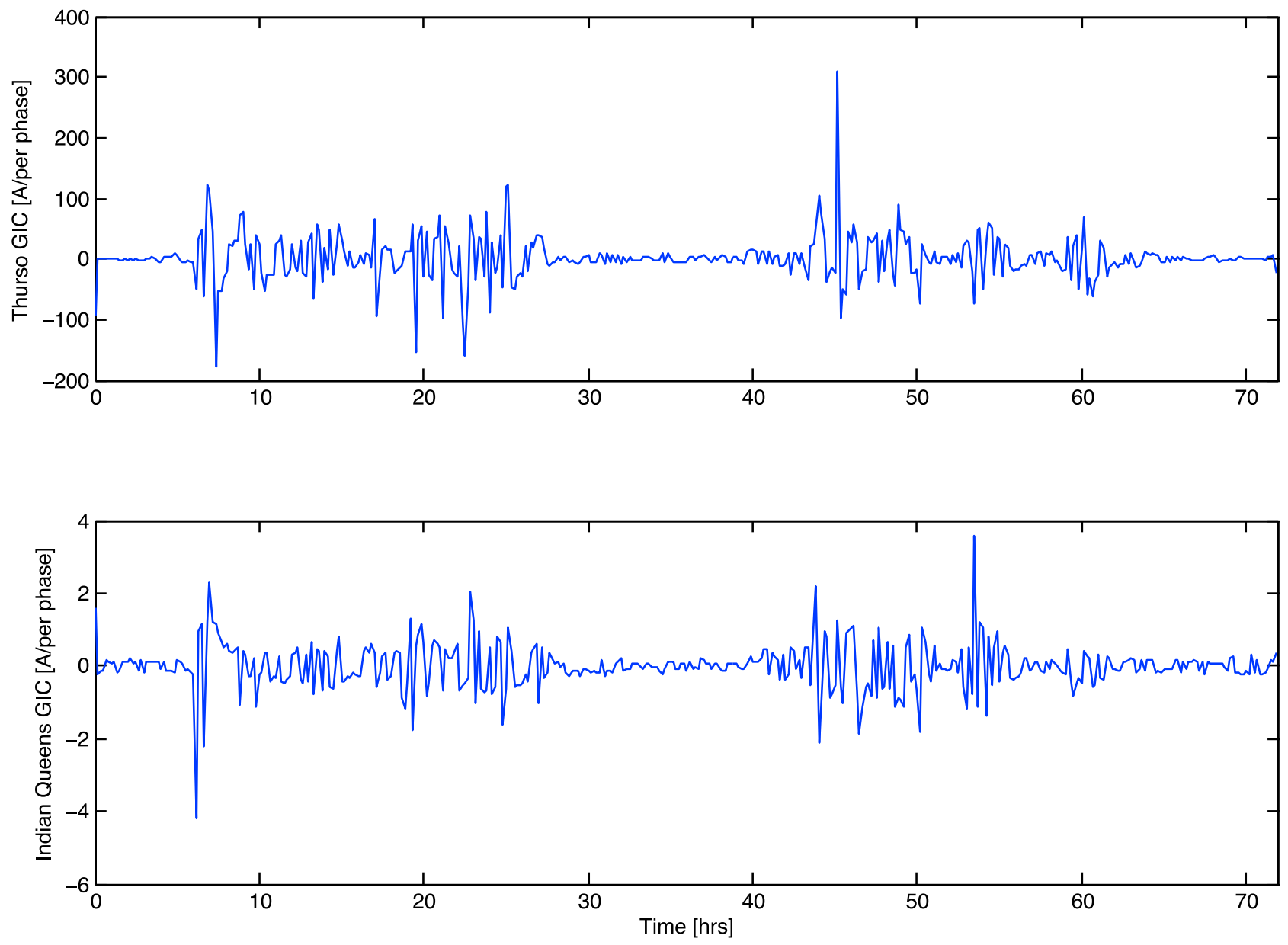

Figure 11. Time series of the flow of GIC at the Thurso (far northern point) and Indian Queens (far southwestern point) nodes in the U.K. grid for the scenario described in Figure 10. One transformer per node is assumed. Currents are per-phase GIC at each node. Only maximum amplitude GIC taken over 10-min windows are shown.

et al., 2006] or by inverting the parameters from GIC and ground magnetic field observations [Pulkkinen et al., 2007]. The linear relation in equation (5) has been shown in numerous studies to hold to a good approximation in many situations of interest [Pulkkinen et al., 2006; Ngwira et al., 2008; Pulkkinen et al., 2010]. Typical values for $(a, b)$ range from 0 to $200 \mathrm{~A} \cdot \mathrm{km} / \mathrm{V}$ [Pulkkinen et al., 2008, and references therein]. For example, using mid-range $a=b=50 \mathrm{~A} \cdot \mathrm{km} / \mathrm{V}$, which we assume to represent sum of GIC over three phases flowing through transformer to the ground, one gets the extreme GIC scenario in Figure 12 for conductive ground at a location above the threshold geomagnetic latitude, i.e., for the scenario in Figure 7b.

\section{Discussion}

[53] In this paper we explored 100-year extreme geoelectric field scenarios by taking into account the key geophysical factors associated with the geomagnetic induction process. More specifically, we derived explicit geoelectric field temporal profiles as a function of ground conductivity structures and geomagnetic latitudes. We also demonstrated how the extreme geoelectric field scenarios can be mapped into GIC. These computed GIC could then be use in further engineering analyses that are needed to quantify the impact of extreme space weather events on conductor systems such as high-voltage power transmission systems.

[54] Although we hope that the work in this paper provides initial input for engineering analyses, it is emphasized that further work is needed to refine and improve the generated scenarios. For example, due to the poor knowledge of global spatial characteristics of the geoelectric field during extreme events, the scenarios presented in this paper apply only on regional scales of the order of 100$1000 \mathrm{~km}$. It is of great interest to expand the scenarios for application in direct global calculations. Also, onedimensional representative ground conductivity models 


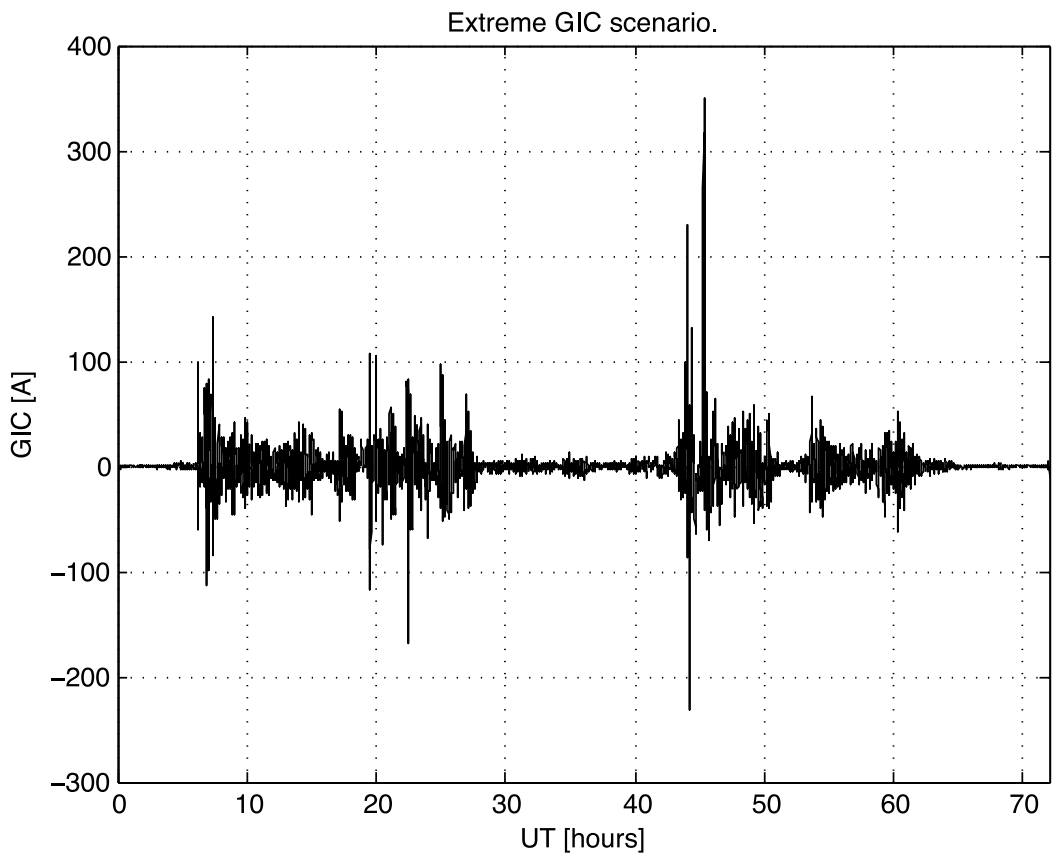

Figure 12. Extreme GIC scenario for conductive ground at locations above the threshold geomagnetic latitude. System parameters $a=b=50 \mathrm{~A} \cdot \mathrm{km} / \mathrm{V}$, which we assume to represent the sum of GIC over three phases flowing through the transformer to the ground, were used to map the geoelectric field into GIC. See the text for details.

were used to account for the varying electromagnetic response of different local geological structures. In principle, if the local ground conductivity is known, the statistics used in this paper can be tailored for specific regions, providing significant refinement for extreme geoelectric field scenarios. It is also of interest to investigate the impact of steep horizontal ground conductivity gradients on the extreme geoelectric field scenarios. For this one would need to utilize a more complex mathematical framework, allowing two- or three-dimensional ground conductivity structures in the calculation of the geoelectric field. We, however, emphasize that effective one-dimensional ground conductivity models applied with the plane wave method is reasonable in many, if not in most, situations.

[55] Perhaps the most critical and still somewhat open question that needs further clarification concerns the dynamics and the location of the identified threshold geomagnetic latitude. Since the geoelectric field amplitude experiences a significant drop across the threshold, the location has significant implications for the extension of global impacts of extreme storms. Detailed studies of historical records of extreme geomagnetic storms and accompanied auroral sightings, as well as physics-based magnetosphere-ionosphere models capturing the key physical processes associated with the low-latitude auroral boundary, may be used to shed further light on the topic. We emphasize that pending further studies on the possible boundary location for 100-year storm events, one should be careful in applying the location in GIC analyses.
[56] Ultimately, only high temporal resolution global geomagnetic recordings for extended time periods provide definitive quantification of likelihoods and spatiotemporal characterization of extreme geomagnetic storm events. Modern 60-s digital recordings have been available only since the early 1980s, which presents obvious difficulties in trying to extract information about 100-year events today. Regular refinement of the statistics derived in this paper should thus be carried out and over time we will be able to quantify more definitively the severity of 100-year geomagnetic storms and their impacts on high-voltage power transmission systems.

[57] Since the major goal of the work at hand is to facilitate further engineering analyses quantifying the impacts of extreme geomagnetic storms on high-voltage transmission systems, the generated extreme geoelectric field scenarios in Figure 7 are publicly available. The digital data can be requested from A. Pulkkinen (pulkkinen@cua. edu; antti.a.pulkkinen@nasa.gov).

\section{Appendix A: Mapping the Ground Geomagnetic Field Into the Geoelectric Field}

[58] Because of the central role of the process in most GIC modeling, we give here a brief overview of the application of the plane wave method to mapping of the ground geomagnetic field fluctuations into the geoelectric field. The method was first formulated by Cagniard [1953] and has since been used extensively in general geomagnetic induction and magnetotelluric studies. The method 
is based on the concept of surface impedance, which is defined as

$$
\tilde{Z}=\mu_{0} \frac{\tilde{E}_{x}}{\tilde{B}_{y}}=-\mu_{0} \frac{\tilde{E}_{y}}{\tilde{B}_{x}},
$$

where $\left(\tilde{E}_{x}, \tilde{E}_{y}\right)$ and $\left(\tilde{B}_{x}, \tilde{B}_{y}\right)$ are the horizontal components of the electric field and magnetic field, respectively, $\mu_{0}$ is the vacuum permeability and tilde indicates quantities in the spectral domain. In geophysical applications of equation (A1) the fields are evaluated at the surface of the Earth. By assuming quasi-static temporal fluctuations, i.e., neglecting the displacement current in Maxwell's equations, and by assuming that the horizontal field gradients vanish, the impedance of the layer $n$ of one-dimensional layered ground can be computed using the recursive formula

$$
\tilde{Z}_{n}=\frac{i \omega \mu_{0}}{\gamma_{n}} \operatorname{coth}\left(\gamma_{n} d_{n}+\operatorname{coth}^{-1}\left(\frac{\gamma_{n}}{i \omega \mu_{0}} \tilde{Z}_{n+1}\right)\right),
$$

where $\gamma_{n}^{2}=i \omega \mu_{0} \sigma_{n}, d_{n}$ is the thickness of the layer $n, \omega$ is the angular frequency of the field fluctuations and $\sigma_{n}$ is the conductivity of the layer $n$. To obtain the surface impedance in equation (A1), one sets $n=1$ in equation (A2) and computes the impedance values recursively starting from the bottom of the modeled ground structure. The bottom layer is assumed infinitely thick.

[59] While the quasi-stationary approximation is valid for geomagnetic induction studies having typical frequencies of temporal field fluctuations below $1 \mathrm{~Hz}$ [Weaver, 1994], the assumption about vanishing horizontal gradients of field fluctuations may seem at first invalid, especially during strong geomagnetic storm conditions. However, Dmitriev and Berdichevsky [1979] showed that the above formulation holds also if the plane wave requirement is relaxed into an assumption about locally $(\sim 100 \mathrm{~km})$ linear variation of the surface magnetic field. The extended validity of the "plane wave" formulation is likely to be one key reason for the success of the method in GIC applications.

[60] The process for mapping the ground geomagnetic field into the geoelectric field is then as follows:

[61] 1. Convert the horizontal ground geomagnetic field into the spectral domain by using the Fourier transform.

[62] 2. Compute the surface impedance using equation (A2).

[63] 3. Compute the spectral domain horizontal geoelectric field using equation (A1).

[64] 4. Convert the spectral domain geoelectric field into the time domain using the inverse Fourier transform.

[65] It is also noted that it follows from the basic properties of the Fourier transform:

$$
\tilde{B}=\frac{1}{i \omega} \frac{d \tilde{B}}{d t}(\omega)
$$

Consequently, computation of the geoelectric field from both the ground geomagnetic field and the time derivative of the geomagnetic field using Equations (A1) and (A2) is a linear operation.
[66] Acknowledgments. This work was carried out in connection with the North American Electric Reliability Corporation Geomagnetic Disturbances Task Force (GMDTF) activity taking place during year 2011. The valuable feedback from the GMDTF community helped greatly to shape our work and to make the all important connection between the geophysics and power engineering sides of the GIC problem. We acknowledge especially R. Lordan (Electric Power Research Institute), D. Watkins (Bonneville Power Administration), L. Marti (Hydro One), M. Hesse (NASA Goddard Space Flight Center), H. Nevanlinna (Finnish Meteorological Institute), A. Viljanen (Finnish Meteorological Institute), R. Pirjola (Finnish Meteorological Institute), and R. Kataoka (Tokyo Institute of Technology) for fruitful discussions and comments that substantially enhanced the quality of our work. Y. Zheng (NASA Goddard Space Flight Center) is acknowledged for help with the study of the location of auroral boundaries during the March 1989 event. We are grateful to S. Silverman for data and discussions on the important question regarding the lowlatitude auroral boundaries. The results presented in this paper rely on data collected at magnetic observatories. We thank the national institutes that support them and INTERMAGNET for promoting high standards of magnetic observatory practice (www.intermagnet.org). The Dst index data was provided by the World Data Center for Geomagnetism, Kyoto, Japan. We thank the institutes who maintain the IMAGE Magnetometer Array that provided the data used in deriving the geoelectric field statistics for this work. A. Viljanen is acknowledged for providing Nurmijärvi Geophysical Observatory data. Geomagnetic field data at Memanbetsu station was provided by Kakioka Magnetic Observatory, Japan Meteorological Agency (KMO-JMA). This paper is published with the permission of the Director of the British Geological Survey. The research leading to these results has received funding from the European Community's Seventh Framework Programme (FP7/2007-2013) under grant agreement 260330

\section{References}

Ahn, B.-H., G. X. Chen, W. Sun, J. W. Gjerloev, Y. Kamide, J. B. Sigwarth, and L. A. Frank (2005), Equatorward expansion of the westward electrojet during magnetically disturbed periods, J. Geophys. Res., 110, A01305, doi:10.1029/2004JA010553.

Albertson, V. D., et al. (1981), Load-flow studies in the presence of geomagnetically-induced currents, IEEE Trans. Power Syst., 100(2), 594-607.

Allen, J., H. Sauer, L. Frank, and P. Reiff (1989), Effects of the March 1989 solar activity, Eos Trans. AGU, 70(46), 1479, doi:10.1029/ $89 \mathrm{EO} 00409$.

Anderson, C. W., L. J. Lanzerotti, and C. G. MacLennan (1974), Outage of the L4 system and the geomagnetic disturbances of 4 August 1972, Bell Syst. Tech. J., 53(9), 1817-1837.

Beamish, D., T. D. G. Clark, E. Clarke, and A. W. P. Thomson (2002), Geomagnetically induced currents in the UK: Geomagnetic variations and surface electric fields, J. Atmos. Sol. Terr. Phys., 64, 1779-1792.

Berdichevsky, M., and M. Zhdanov (1984), Advanced Theory of Deep Geomagnetic Sounding, 408 pp., Elsevier Sci., Amsterdam, Netherlands.

Bolduc, L. (2002), GIC observations and studies in the Hydro-Quebec power system, J. Atmos. Sol. Terr. Phys., 64, 1793-1802.

Borovsky, J. E., and M. H. Denton (2006), Differences between CMEdriven storms and CIR-driven storms, J. Geophys. Res., 111, A07S08, doi:10.1029/2005JA011447.

Boteler, D. (1997), Distributed source transmission line theory for electromagnetic induction studies, in Supplement of the Proceedings of the 12th International Zurich Symposium and Technical Exhibition on Electromagnetic Compatibility, pp. 401-408, Swiss Fed. Inst. of Tech., Zurich, Switzerland.

Boteler, D. H. (2001), Assessment of geomagnetic hazard to power systems in Canada, Nat. Hazards, 23, 101-120.

Boteler, D. H. (2006), The super storms of August/September 1859 and their effects on the telegraph system, Adv. Space Res., 38, 159-172.

Cagniard, L. (1953), Basic theory of the magneto-telluric method of geophysical prospecting, Geophysics, 18(3), 605. 
Campbell, W. C. (1980), Observation of electric currents in the Alaska oil pipeline resulting from auroral electrojet current sources, Geophys. J. R. Astron. Soc., 61, 437-449.

Cliver, E. W., and L. Svalgaard (2004), The 1859 solar-terrestrial disturbance and the current limits of extreme space weather activity, Sol. Phys., 224, 407-422.

Dmitriev, V. I., and M. N. Berdichevsky (1979), The fundamental model of magnetotelluric sounding, IEEE Proc., 67, 1034-1044.

Gaunt, C. T., and G. Coetzee (2007), Transformer failures in regions incorrectly considered to have low GIC-risk, in Power Tech, 2007 IEEE Lausanne: Proceedings, pp. 807-812, Inst. of Elec. and Elec. Eng., Piscataway, N. J.

Grossi, P., and H. Kunreuther (Eds.) (2005), Catastrophe Modeling: A New Approach to Managing Risk, Springer Sci., Boston, Mass.

Huttunen, K. E. J., S. P. Kilpua, A. Pulkkinen, A. Viljanen, and E. Tanskanen (2008), Solar wind drivers of large geomagnetically induced currents during the solar cycle 23, Space Weather, 6, S10002, doi:10.1029/2007SW000374.

Kappenman, J. G. (1996), Geomagnetic storms and their impact on power systems, IEEE Power Eng. Rev., 16(5), 5-8.

Kappenman, J. G. (2003), Storm sudden commencement events and the associated geomagnetically induced current risks to groundbased systems at low-latitude and midlatitude locations, Space Weather, 1(3), 1016, doi:10.1029/2003SW000009.

Kappenman, J. G. (2006), Great geomagnetic storms and extreme impulsive geomagnetic field disturbance events $Đ$ An analysis of observational evidence including the great storm of May 1921, Adv. Space Res., 38, 188-199.

Kataoka, R., and A. Pulkkinen (2008), Geomagnetically induced currents during intense storms driven by coronal mass ejections and corotating interacting regions, J. Geophys. Res., 113, A03S12, doi:10.1029/2007JA012487.

Langlois, P., L. Bolduc, and M. C. Chouteau (1996), Probability of occurrence of geomagnetic storms based on a study of the distribution of the electric field amplitudes measured in Abitibi, Québec, in 1993-1994, J. Geomagn. Geoelectr., 48, 1033-1041.

Lehtinen, M., and R. Pirjola (1985), Currents produced in earthed conductor networks by geomagnetically-induced electric fields, Ann. Geophys., 3(4), 479-484.

Loomis, E. (1860), The great auroral exhibition of August 28th to September 4th, 1859, Am. J. Sci., 29, 386-399.

Lühr, H., S. Maus, and M. Rother (2004), Noon-time equatorial electrojet: Its spatial features as determined by the CHAMP satellite, J. Geophys. Res., 109, A01306, doi:10.1029/2002JA009656.

Molinski, T. (2002), Why utilities respect geomagnetically induced currents, J. Atmos. Sol. Terr. Phys., 64, 1765-1778.

Muir-Wood, R., and P. Grossi (2008), The catastrophe modeling response to Hurricane Katrina, in Climate Extremes and Society, edited by H. F. Diaz and R. J. Murnane, pp. 296-319, Cambridge Univ. Press, Cambridge, U. K.

National Research Council (2008), Severe Space Weather Events: Understanding Societal and Economic Impacts-A Workshop Report, Natl. Acad. Press, Washington, D. C.

Nevanlinna, H. (2006), A study on the great geomagnetic storm of 1859: Comparisons with other storms in the 19th century, Adv. Space Res., $38,180-187$.

Nevanlinna, H. (2008), On geomagnetic variations during the AugustSeptember storms of 1859, Adv. Space Res., 42, 171-180.

Ngwira, C. M., A. Pulkkinen, L.-A. McKinnell, and P. J. Cilliers (2008), Improved modeling of geomagnetically induced currents in the South African power network, Space Weather, 6, S11004, doi:10.1029/2008SW000408.

North American Electric Reliability Corporation and the U.S. Department of Energy (2010), High-Impact, Low-Frequency Event Risk to the North American Bulk Power System: A Jointly-Commissioned Summary Report of the North American Electric Reliability Corporation and the U.S. Department of Energy's November 2009 Workshop, 120 pp., North Am. Elec. Reliab. Corp., Washington, D. C. [Available at www.nerc. com/files/HILF.pdf.]

Ohtani, S.-I., R. Fujii, M. Hesse, and R. L. Lysak (Eds.) (2000), Magnetospheric Current Systems, AGU Geophys. Monogr. 118, AGU, Washington, D. C.

Pulkkinen, A. (2007), Spatiotemporal characteristics of the ground electromagnetic field fluctuations in the auroral region and implications on the predictability of geomagnetically induced currents, in Space Weather: Research Towards Applications in Europe, Astrophys. Space Sci. Lib., vol. 344, edited by J. Lilensten, 332 pp., Springer, Dordrecht, Netherlands.

Pulkkinen, A., and R. Kataoka (2006), S-transform view of geomagnetically induced currents during geomagnetic superstorms, Geophys. Res. Lett., 33, L12108, doi:10.1029/2006GL025822.

Pulkkinen, A., A. Thomson, E. Clarke, and A. McKay (2003), April 2000 geomagnetic storm: Ionospheric drivers of large geomagnetically induced currents, Ann. Geophys., 21, 709-717.

Pulkkinen, A., S. Lindahl, A. Viljanen, and R. Pirjola (2005), Geomagnetic storm of 29-31 October 2003: Geomagnetically induced currents and their relation to problems in the Swedish high-voltage power transmission system, Space Weather, 3, S08C03, doi:10.1029/ 2004SW000123.

Pulkkinen, A., A. Viljanen, and R. Pirjola (2006), Estimation of geomagnetically induced current levels from different input data, Space Weather, 4, S08005, doi:10.1029/2006SW000229.

Pulkkinen, A., R. Pirjola, and A. Viljanen (2007), Determination of ground conductivity and system parameters for optimal modeling of geomagnetically induced current flow in technological systems, Earth Planets Space, 59, 999-1006.

Pulkkinen, A., R. Pirjola, and A. Viljanen (2008), Statistics of extreme geomagnetically induced current events, Space Weather, 6, S07001, doi:10.1029/2008SW000388.

Pulkkinen, A., R. Kataoka, S. Watari, and M. Ichiki (2010), Modeling geomagnetically induced currents in Hokkaido, Japan, Adv. Space Res., 46, 1087-1093.

Silverman, S. M. (2006), Comparison of the aurora of September 1/2, 1859, with other great auroras, Adv. Space Res., 38, 136-144.

Silverman, S. M., and E. W. Cliver (2001), Low-latitude auroras: The magnetic storm of 14-15 May 1921, J. Atmos. Sol. Terr. Phys., 63, 523-535.

Siscoe, G., N. U. Crooker, and C. R. Clauer (2006), Dst of the Carrington storm of 1859, Adv. Space Res., 38, 173-179.

Thomson, A. W. P., A. J. McKay, E. Clarke, and S. J. Reay (2005), Surface electric fields and geomagnetically induced currents in the Scottish Power grid during the 30 October 2003 geomagnetic storm, Space Weather, 3, S11002, doi:10.1029/2005SW000156.

Thomson, A., S. Reay, and E. Dawson (2011), Quantifying extreme behavior in geomagnetic activity, Space Weather, 9, S10001, doi:10.1029/2011SW000696.

Trichtchenko, L., and D. H. Boteler (2004), Modeling geomagnetically induced currents using geomagnetic indices and data, IEEE Trans. Plasma Sci., 32(4), 1459-1467.

Tsubouchi, K., and Y. Omura (2007), Long-term occurrence probabilities of intense geomagnetic storm events, Space Weather, 5, S12003, doi:10.1029/2007SW000329.

Tsurutani, B. T., W. D. Gonzalez, G. S. Lakhina, and S. Alex (2003), The extreme magnetic storm of 1-2 September 1859, J. Geophys. Res., 108(A7), 1268, doi:10.1029/2002JA009504.

Turnbull, K. (2011), A study of geomagnetically induced currents in the UK national grid, PhD thesis, Univ. of Lancaster, Lancaster, U. K.

Viljanen, A., H. Nevanlinna, K. Pajunpää, and A. Pulkkinen (2001), Time derivative of the horizontal geomagnetic field as an activity indicator, Ann. Geophys., 19, 1107-1118.

Viljanen, A., A. Pulkkinen, R. Pirjola, K. Pajunpää, P. Posio, and A. Koistinen (2006a), Recordings of geomagnetically induced currents and a now casting service of the Finnish natural gas pipeline system, Space Weather, 4, S10004, doi:10.1029/2006SW000234.

Viljanen, A., E. I. Tanskanen, and A. Pulkkinen (2006b), Relation between substorm characteristics and rapid temporal variations of the ground magnetic field, Ann. Geophys., 24, 725-733.

Wait, J. R. (1970), Electromagnetic Waves in Stratified Media, 2nd ed., 608 pp., Pergamon, Oxford, U. K.

Weaver, J. T. (1994), Mathematical Methods for Geo-electromagnetic Induction, 316 pp., Wiley, New York.

Weigel, R. S., and D. N. Baker (2003), Probability distribution invariance of 1-minute auroral-zone geomagnetic field fluctuations, Geophys. Res. Lett., 30(23), 2193, doi:10.1029/2003GL018470.

Wik, M., A. Viljanen, R. Pirjola, A. Pulkkinen, P. Wintoft, and H. Lundstedt (2008), Calculation of geomagnetically induced 
currents in the $400 \mathrm{kV}$ power grid in southern Sweden, Space Weather, 6, S07005, doi:10.1029/2007SW000343.

Yokoyama, N., Y. Kamide, and H. Miyaoka (1998), The size of auroral belt during magnetic storms, Ann. Geophys., 16, 566-573.

C. Beggan and A. W. P. Thomson, British Geological Survey, Murchison House, West Mains Rd., Edinburgh EH9 3LA, UK.
E. Bernabeu, Dominion Virginia Power, 120 Tredegar St., Richmond, VA 23219, USA.

J. Eichner, Munich Re, Königinstr. 107, 80802 Munich, Germany.

A. Pulkkinen, NASA Goddard Space Flight Center, Code 674, Greenbelt, MD 20771, USA. 